\title{
Selectively Inhibiting the Median Preoptic Nucleus Attenuates Angiotensin II and Hyperosmotic- Induced Drinking Behavior and Vasopressin Release in Adult Male Rats
}

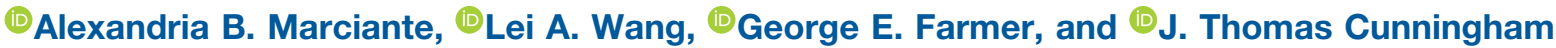

https://doi.org/10.1523/ENEURO.0473-18.2019

Department of Physiology and Anatomy, University of North Texas Health Science Center, Fort Worth, TX 76107

\section{Visual Abstract}

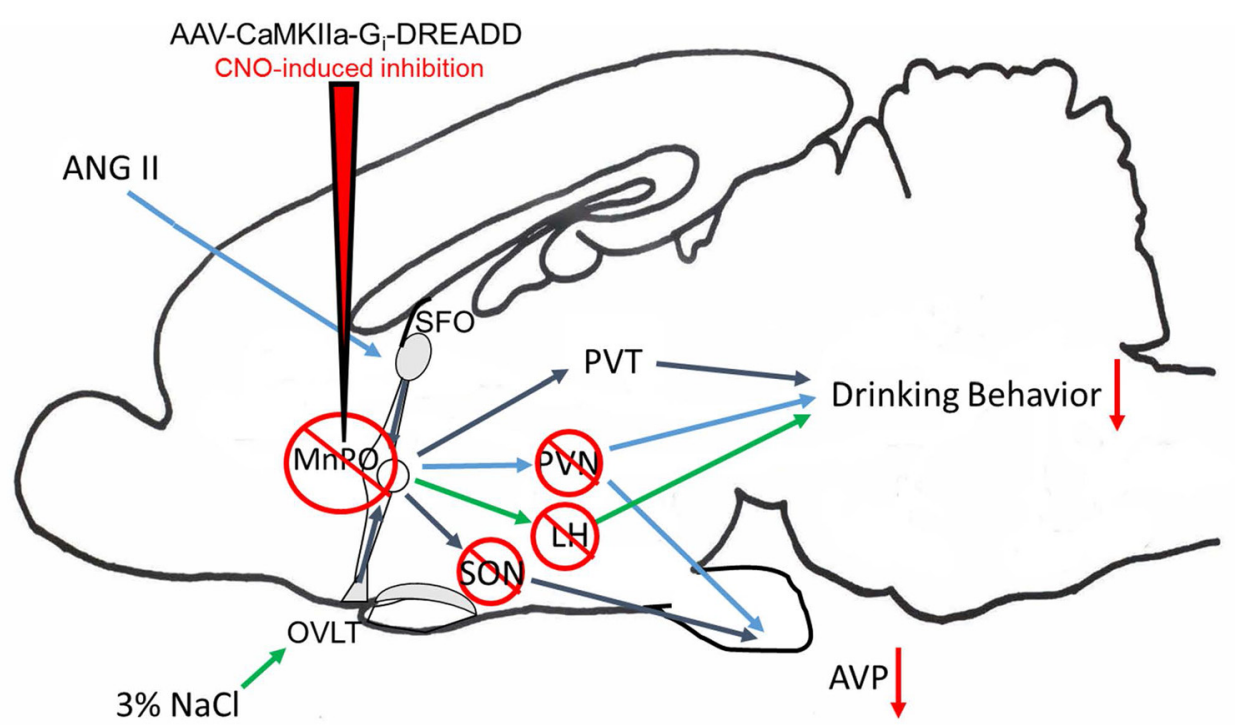

The median preoptic nucleus (MnPO) is a putative integrative region that contributes to body fluid balance. Activation of the MnPO can influence thirst, but it is not clear how these responses are linked to body fluid homeostasis. We used designer receptors exclusively activated by designer drugs (DREADDs) to determine the

\section{Significance Statement}

The median preoptic nucleus $(\mathrm{MnPO})$ is an important regulatory center that influences thirst, cardiovascular and neuroendocrine function. Activation of different MnPO neuronal populations can inhibit or stimulate water intake. However, the role of the $\mathrm{MnPO}$ and its pathway-specific projections during angiotensin II (ANG II) and hyperosmotic challenges still have not yet been fully elucidated. These studies directly address this by using designer receptors exclusively activated by designer drugs (DREADDs) to acutely and selectively inhibit pathway-specific MnPO neurons, and uses techniques that measure changes at the protein, neuronal, and overall physiologic and behavioral level. More importantly, we have been able to demonstrate that physiologic challenges related to extracellular (ANG II) or cellular (hypertonic saline) dehydration activate MnPO neurons that may project to different parts of the hypothalamus. 
role of the MnPO in drinking behavior and vasopressin release in response to peripheral angiotensin II (ANG II) or $3 \%$ hypertonic saline (3\% HTN) in adult male Sprague Dawley rats $(250-300 \mathrm{~g})$. Rats were anesthetized with isoflurane and stereotaxically injected with an inhibitory DREADD (rAAV5-CaMKIla-hM4D $\left(\mathrm{G}_{\mathrm{i}}\right)-\mathrm{mCherry}$ ) or control (rAAV5-CaMKIla-mCherry) virus in the MnPO. After two weeks' recovery, a subset of rats was used for extracellular recordings to verify functional effects of ANG II or hyperosmotic challenges in MnPO slice preparations. Remaining rats were used in drinking behavior studies. Each rat was administered either $10 \mathrm{mg} / \mathrm{kg}$ of exogenous clozapine-N-oxide (CNO) to inhibit DREADD-expressing cells or vehicle intraperitoneal followed by a test treatment with either 2-mg/kg ANG II or 3\% HTN (1 ml/100-g bw, s.c.), twice per week for two separate treatment weeks. CNO-induced inhibition during either test treatment significantly attenuated drinking responses compared to vehicle treatments and controls. Brain tissue processed for cFos immunohistochemistry showed decreased expression with $\mathrm{CNO}$-induced inhibition during either test treatment in the $\mathrm{MnPO}$ and downstream nuclei compared to controls. CNO-mediated inhibition significantly attenuated treatment-induced increases in plasma vasopressin compared to controls. The results indicate inhibition of CaMKlla-expressing MnPO neurons significantly reduces drinking and vasopressin release in response to ANG II or hyperosmotic challenge.

Key words: ADH; Fos; osmoreceptor; thirst

\section{Introduction}

The median preoptic nucleus (MnPO) is a midline nucleus that is part of the lamina terminalis, or anteroventral 3rd ventricle (AV3V) region. It plays an important role in receiving and integrating signals related to homeostasis and further propagating information to the hypothalamus (McKinley et al., 2015). Neurons in the MnPO contribute to the central regulation of sleep, core temperature, body fluid balance, hormone release, and autonomic function (McKinley et al., 2015). These studies focus on the role of the $\mathrm{MnPO}$ in regulating body fluid homeostasis through drinking behavior and vasopressin release.

The MnPO receives projections from two circumventricular organs (CVOs), the organum vasculosum of the lamina terminalis (OVLT) and the subfornical organ (SFO), that lie just ventral and dorsal to the MnPO along the anterior wall of the third ventricle, respectively. CVOs respond to fluctuations in plasma osmolality and other humoral factors, like angiotensin II (ANG II; Johnson et al., 1996). The OVLT and SFO project to several hypothalamic regions that contribute to homeostasis through behavioral, endocrine, and autonomic responses. These pathways include, but are not limited to, the supraoptic nucleus (SON), paraventricular nucleus of the hypothalamus (PVN), and the MnPO (Miselis, 1982; McKinley et al., 2004). The MnPO has reciprocal connections with the OVLT and SFO and also projects to the SON and PVN

Received December 6, 2018; accepted February 26, 2019; First published March 7, 2019.

The authors declare no competing financial interests.

Author contributions: A.B.M., L.A.W., G.E.F., and J.T.C. designed research; A.B.M., L.A.W., and G.E.F. performed research; A.B.M., L.A.W., and G.E.F. analyzed data; A.B.M. and J.T.C. wrote the paper.

This work was supported by NHLBI Grants R01 HL142341, P01 HL088052, and T32 AG020494.

Acknowledgements: We thank the technical assistance of J.T. Little, M. Bachelor, and J. Kiehlbauch.

Correspondence should be addressed to J. Thomas Cunningham at tom.cunningham@unthsc.edu.

https://doi.org/10.1523/ENEURO.0473-18.2019

Copyright (C) 2019 Marciante et al.

This is an open-access article distributed under the terms of the Creative Commons Attribution 4.0 International license, which permits unrestricted use, distribution and reproduction in any medium provided that the original work is properly attributed.
(McKinley et al., 2015). Studies have also shown that the $\mathrm{MnPO}$ has projections to the lateral hypothalamus (LH) and paraventricular thalamus (PVT) to regulate drinking behavior (Leib et al., 2017). The role, however, of the $\mathrm{MnPO}$ and its relationship to specific challenges regarding body fluid homeostasis are not completely understood.

Body fluid homeostasis involves orchestrated physiologic responses to cellular or extracellular dehydration (Fitzsimons, 1972; Adler and Verbalis, 2006). Cellular dehydration results from changes in body fluid solute content that is detected by osmoreceptors the in peripheral and central nervous system (Bourque, 2008). Extracellular dehydration is related to changes in body fluid volume that influence several hormonal and neural systems (Mecawi Ade et al., 2015; Dampney, 2016). Changes in the function of these systems contribute to several pathophysiological states (Adler and Verbalis, 2006). The contribution of the CNS to this physiology and pathophysiology has been the subject of previous investigations.

Lesions of the rat AV3V region produce a lifethreatening adipsia (Johnson et al., 1996). If properly maintained, however, rats can recover some spontaneous water intake but do not respond to experimental challenges that mimic aspects of homeostatic thirst, such as ANG II or osmotic stimulation (Johnson et al., 1996).

Studies using electrolytic or chemical lesions of the $\mathrm{MnPO}$ have produced conflicting results. For example, electrolytic lesions of the MnPO are reported to increase baseline drinking and prevent vasopressin release (Gardiner and Stricker, 1985; Gardiner et al., 1985). Excitotoxin lesions of the MnPO inhibit experimentally-induced drinking behavior without affecting basal water intake (Cunningham et al., 1992).

A recent study by Allen et al. (2017) used a new technique involving the induction of Fos in neurons, a measurement for acute neuronal activation, co-transfected with TRAP creating an in-frame fusion to characterize $\mathrm{MnPO}$ neurons based on their activation by water deprivation or changes in body temperature. This study showed that putative thirst-related neurons in the MnPO are different from those that respond to body temperature. However, water deprivation is a complex, progres- 
sive physiologic challenge characterized by increased plasma osmolality, hypovolemia, and activation of the renin-angiotensin system. It is not clear whether MnPO neuronal activation associated with water deprivation is segregated by the physiologic stimulus or stimuli associated with water deprivation. The MnPO also contributes to other aspects of body fluid homeostasis in addition to thirst.

Recently developed chemogenetic and optogenetic techniques have been used to study neuronal circuitry responsible for mediating thirst in the lamina terminalis. A study by Augustine et al. (2018) recently used these approaches to characterize MnPO neurons that stimulate or inhibit thirst. Their results provide important information about the neurochemical phenotype of these two cell populations, however, the physiologic stimuli that regulate the activity of these cell types is not clear.

In the current study, AAV vectors with CaMKIla promoters were used to express designer receptors exclusively activated by designer drugs (DREADDs) in the MnPO to inhibit CaMKIla-expressing neurons with clozapine-Noxide (CNO). Conscious rats were administered CNO during protocols that simulate extracellular (ANG II) or intracellular dehydration (hypertonic saline) to assess the role of the MnPO in behavioral and neuroendocrine responses to specific homeostatic challenges.

\section{Materials and Methods}

\section{Animals}

Adult male Sprague Dawley rats (250-300 g bw; Charles River Laboratories) were used for experiments. All rats were individually housed in a temperature-controlled $\left(25^{\circ} \mathrm{C}\right)$ room on a $12 / 12 \mathrm{~h}$ light/dark cycle with light onset at 7 A.M. Food and water was available ad libitum except on the day of perfusions. Rats were weighed daily and their food and water intake monitored. Experiments were performed according to the National Institutes of Health guide for the care and use of laboratory animals and the Institutional Animal Care and Use Committee.

\section{Microinjection surgeries}

Rats were anesthetized with $2 \%$ isoflurane and received stereotaxic microinjections of the inhibitory (AAV5CaMKIla-hM4D $\left(G_{i}\right)-m$ Cherry) Cre-independent DREADD or control (AAV5-CaMKIla-mCherry-Cre) virus (both from the UNC VectorCore) into the MnPO (microinjector angled at $8^{\circ}$ from medial to lateral to avoid the septum, $0.0 \mathrm{~mm}$ anterior, $0.9 \mathrm{~mm}$ lateral, $-6.7 \mathrm{~mm}$ ventral from bregma). A burr hole was then drilled at the measured site and a 30-gauge stainless steel injection needle was lowered to the MnPO, where $200-300 \mathrm{nl}$ of AAV was delivered at a rate of $200 \mathrm{nl} / \mathrm{min}$. The injector was connected to a Hamilton 5 - $\mu$ l syringe (\#84851, Hamilton) by calibrated polyethylene tubing that was used to determine the injection volume. The injector remained in place for $5 \mathrm{~min}$ to allow for absorption and then slowly withdrawn. Gel foam was packed in to the drilled hole in the cranium. Absorbable antibiotic suture was used to close the incision site and minimize post-surgical infection. Each rat was given carprofen (Rimadyl, Bio-Serv, $1 \mathrm{mg}$ ) orally to minimize pain following surgery. Rats were allowed time for recovery and viral transfection for two weeks.

\section{In situ hybridization}

In situ hybridization experiments were performed to characterize the neuronal phenotype of CaMKIla-positive $\mathrm{MnPO}$ neurons transfected by the control virus. After the two-week recovery period, rats were anesthetized using $100-\mathrm{mg} / \mathrm{kg}$ inactin (Sigma-Aldrich) intraperitoneally and transcardially flushed first with RNase-free PBS. Rats were then perfused using $4 \%$ paraformaldehyde (PFA). Brains were dehydrated in RNase-free $30 \%$ sucrose. Twenty $\mu \mathrm{m}$ coronal sections of each brain were cut using a cryostat (Leica). Six sets of serial MnPO sections were collected in RNase-free PBS, mounted on to glass microscope slides, and left at room temperature overnight to dehydrate. Slides were then stored at $-80^{\circ} \mathrm{C}$ until used for in situ hybridization experiments. In situ hybridization was performed for vesicular glutamate transporter 2, or vGLUT2 (RNAScope, Advanced Cell Diagnostics Inc.) using a previously established protocol (Smith et al., 2014).

\section{Electrophysiology}

\section{Slice preparation}

Hypothalamic slices containing the MnPO were prepared as previously described (Farmer et al., 2018). Experimental animals were anesthetized with isoflurane and decapitated. Coronal slices $(300 \mu \mathrm{m})$ containing the $\mathrm{MnPO}$ were cut using a Microslicer DTK Zero 1 (Ted Pella, Inc.) in ice cold $\left(0-1^{\circ} \mathrm{C}\right)$, oxygenated $\left(95 \% \mathrm{O}_{2}, 5 \% \mathrm{CO}_{2}\right)$ cutting solution consisting of $3.0 \mathrm{mM} \mathrm{KCl}, 1.0 \mathrm{mM} \mathrm{MgCl}_{2}-$ $6 \mathrm{H}_{2} \mathrm{O}, 2.0 \mathrm{mM} \mathrm{CaCl}_{2}, 2.0 \mathrm{mM} \mathrm{MgSO}_{4}, 1.25 \mathrm{mM} \mathrm{NaH}_{2} \mathrm{PO}_{4}$, $26 \mathrm{mM} \mathrm{NaHCO}_{3}, 10 \mathrm{mM}$ D-glucose, and $206 \mathrm{mM}$ sucrose (300 mOsm, pH 7.4). Slices were incubated at room temperature in oxygenated $\left(95 \% \mathrm{O}_{2}, 5 \% \mathrm{CO}_{2}\right)$ artificial CSF (aCSF) containing $126 \mathrm{mM} \mathrm{NaCl}, 3.0 \mathrm{mM} \mathrm{KCl}, 2.0 \mathrm{mM}$ $\mathrm{CaCl}_{2}, 2.0 \mathrm{mM} \mathrm{MgSO}{ }_{4}, 1.25 \mathrm{mM} \mathrm{NaH} \mathrm{PO}_{4}, 26 \mathrm{mM}$ $\mathrm{NaHCO}_{3}$, and $10 \mathrm{mM}$ D-glucose (300 mOsm, pH 7.4) for a minimum of $1 \mathrm{~h}$ before recording.

\section{Electrophysiology protocols}

Slices containing the MnPO were transferred to a submersion recording chamber and superfused with aCSF $\left(31 \pm 1^{\circ} \mathrm{C}\right)$. Slices were visualized using an upright epifluorescent microscope (BX50WI, Olympus) with differential interference contrast optics.

Whole-cell (intracellular) recordings were performed in current clamp mode and conducted to measure whether CNO and DREADD-induced inhibition caused off-target effects that would influence the local circuitry. Recordings were obtained using borosilicate glass micropipettes (3-8 $\mathrm{M} \Omega$ ). The internal filling solution consisted of $145 \mathrm{mM}$ K-gluconate, $10 \mathrm{mM}$ HEPES, $1.0 \mathrm{mM}$ EGTA, $2.0 \mathrm{mM}$ $\mathrm{Na}_{2}$ ATP, and 0.4 mM NaGTP (300 mOsm, pH 7.2). A tight gigaohm seal on MnPO neurons were made and had an access resistance of $<25 \mathrm{M} \Omega$. Neurons were slightly depolarized with current injection (2-5 pA) to generate a regular spiking activity (range, -50 to $-40 \mathrm{mV}$ ), as previously described (Grob et al., 2004). Loose patch voltage clamp (extracellular) recordings were obtained using borosilicate glass micropipettes (1-3 $\mathrm{M} \Omega$ ) containing 
aCSF as the internal solution. Voltage was clamped at 0 $\mathrm{mV}$ to measure changes in current.

Electrophysiological signals (voltage and current) were amplified and digitized using Multiclamp 200B and Digidata $1440 \mathrm{~A}$, respectively (Molecular Devices). Signals were filtered at $2 \mathrm{kHz}$ and digitized at $10 \mathrm{kHz}$. Recordings from $\mathrm{MnPO}$ neurons were made by targeting both mCherry-expressing and non mCherry-expressing neurons in slices prepared from rats injected with the AAV. Electrophysiological signals were analyzed using 10-s bins.

In the first set of experiments, intracellular or extracellular recordings were performed on MnPO neurons. Baseline membrane potential (for intracellular recordings) or action potential firing (for extracellular recordings) was recorded for $5 \mathrm{~min}$. Then, CNO $(10 \mu \mathrm{M})$ was focally applied for $10 \mathrm{~s}$ using a Pico spritzer (8 psi) with a patch pipette containing the drug placed 150-200 $\mu \mathrm{m}$ upstream of the recording electrode followed by an additional 10 min of recording. CNO (Tocris) was dissolved in DMSO and diluted in aCSF to final concentration of $10 \mu \mathrm{M}$ $(<0.01 \%$ DMSO).

In the ANG II experiments, baseline action potential firing was recorded for $5 \mathrm{~min}$ in either an aCSF bath solution containing CNO (500 nM) or aCSF alone (for $G_{i}$ DREADD-labeled neuronal controls). Then, ANG II (10 $\mu \mathrm{M})$ was focally applied for $10 \mathrm{~s}$ using a Pico spritzer (8 psi), as in the first set of electrophysiology experiments, followed by $10 \mathrm{~min}$ of recording. ANG II (A9525, Sigma-Aldrich) was dissolved and diluted in aCSF to a final concentration of $10 \mu \mathrm{M}$.

In the hyperosmotic challenge experiments, baseline action potential firing was recorded for $5 \mathrm{~min}$ in an aCSF bath solution containing CNO $(500 \mathrm{nM})$. Then for $2 \mathrm{~min}$, the bath solution was switched to CNO (500 nM) in hypertonic aCSF (HTN-aCSF; 330 mOsm) followed by 2 min of bath application of HTN-aCSF (330 mOsm). For the remaining $5 \mathrm{~min}$ of recording, aCSF was bath applied. Bath application of hyperosmotic solution was used instead of focal application because it allowed for a more exact control of the extracellular $\mathrm{NaCl}$ concentration that would not be possible with focal application. To control for $G_{i}$ DREADD-labeled neuronal activity, experiments were performed following the same time course, however the protocol was $5 \mathrm{~min}$ in aCSF bath, 4 min in HTN-aCSF (330 mOsm), then 5-min aCSF bath. Additional $\mathrm{NaCl}$ was dissolved in aCSF to raise osmolality to $330 \mathrm{mOsm}$.

\section{Drinking study, ANG II}

Before microinjections, rats were pretested with subcutaneous injection of ANG II twice, separated by $48-72 \mathrm{~h}$, to determine individual drinking response to peripherally administered ANG II (consume $\geq 5 \mathrm{ml}$ of water over the course of $3 \mathrm{~h}$ ). Rats that responded were then microinjected with either the $G_{i}$ DREADD or control virus and allowed recovery for two weeks. For each test, rats were intraperitoneally injected with $10-\mathrm{mg} / \mathrm{kg} \mathrm{CNO}$ (dissolved in DMSO and diluted to the working concentration with $0.9 \%$ saline) or vehicle for CNO, VEH (DMSO and $0.9 \%$ saline in 1:4 ratio). Thirty minutes later, rats were admin- istered ANG || $(2 \mathrm{mg} / \mathrm{kg}$, s.c., diluted to the working concentration in $0.9 \%$ saline) or the same volume of $0.9 \%$ saline. Water consumption was measured over the course of $3 \mathrm{~h}$ from the time ANG II was administered to measure drinking response and duration of CNO-induced inhibition. The substances injected for each test were administered in a randomized counter balanced order. The tests were separated by $48-72 \mathrm{~h}$ and were repeated two and three weeks after microinjection surgeries.

\section{Drinking study, $3 \%$ hypertonic saline ( $3 \%$ HTN)}

A separate group of rats was used for drinking studies with $3 \%$ HTN. Rats were pretested consistent with those in the ANG II drinking study to determine individual drinking response to peripherally administered $3 \%$ HTN (consume $\geq 5 \mathrm{ml}$ of water over the course of $3 \mathrm{~h}$ ). Rats that responded were microinjected with the $G_{i}$ DREADD or control virus and allowed recovery for two weeks. After the recovery period, animals were injected intraperitoneally with CNO or VEH and $3 \%$ HTN $(1 \mathrm{ml} / 100-\mathrm{g} \mathrm{bw})$ or the same volume of $0.9 \%$ saline. Study design and duration was consistent with the ANG II drinking study.

\section{Perfusions, tissue and body fluid collection}

After the final treatment week for each drinking study, rats were administered intraperitoneally $\mathrm{CNO}$ and subcutaneously ANG II or 3\% HTN, and denied food and water access the following $90 \mathrm{~min}$. Animals were then anesthetized using $100-\mathrm{mg} / \mathrm{kg}$ inactin (Sigma-Aldrich) intraperitoneally. Blood was collected by cardiac puncture $(3 \mathrm{ml})$ and transferred to an EDTA vacutainer containing $100 \mu \mathrm{l}$ of aprotinin (catalog \#RK-APRO, Phoenix Pharmaceuticals, Inc.) per milliliter of blood (300 $\mu$ l total) immediately preceding the perfusion to measure plasma AVP and osmolality. Rats were transcardially flushed first with PBS and then perfused using 4\% PFA. Brain tissue was fixed overnight in 4\% PFA before being dehydrated in 30\% sucrose.

\section{Arginine vasopressin}

Plasma AVP concentrations were measured by specific EIA (Phoenix Pharmaceuticals, Inc.) following peptide extraction, as recommended by the manufacturer (catalog \#EK-065-07, Phoenix Pharmaceuticals, Inc.). The volume of plasma used was $500 \mu \mathrm{l}$ per sample for peptide extraction. A total of $100 \mu \mathrm{l}$ from each sample was recovered from the extraction and assayed in duplicate $(50 \mu \mathrm{l}$ assayed). The total peptide concentration of each sample was calculated according to the directions provided by the manufacturer of the extraction kit. The intraassay and interassay coefficients of variation averaged $<10 \%$ and $<15 \%$, respectively, as provided by the manufacturer (Phoenix Pharmaceuticals, Inc.).

\section{Immunohistochemistry}

A total of $40-\mu \mathrm{m}$ coronal sections of each previously perfused brain were cut using a cryostat. Three sets of serial sections were separately collected in cryoprotectant (Hoffman et al., 1992) and stored at $-20^{\circ} \mathrm{C}$ until they were processed for immunohistochemistry. Separate sets of serial sections from brains injected with DREADD 


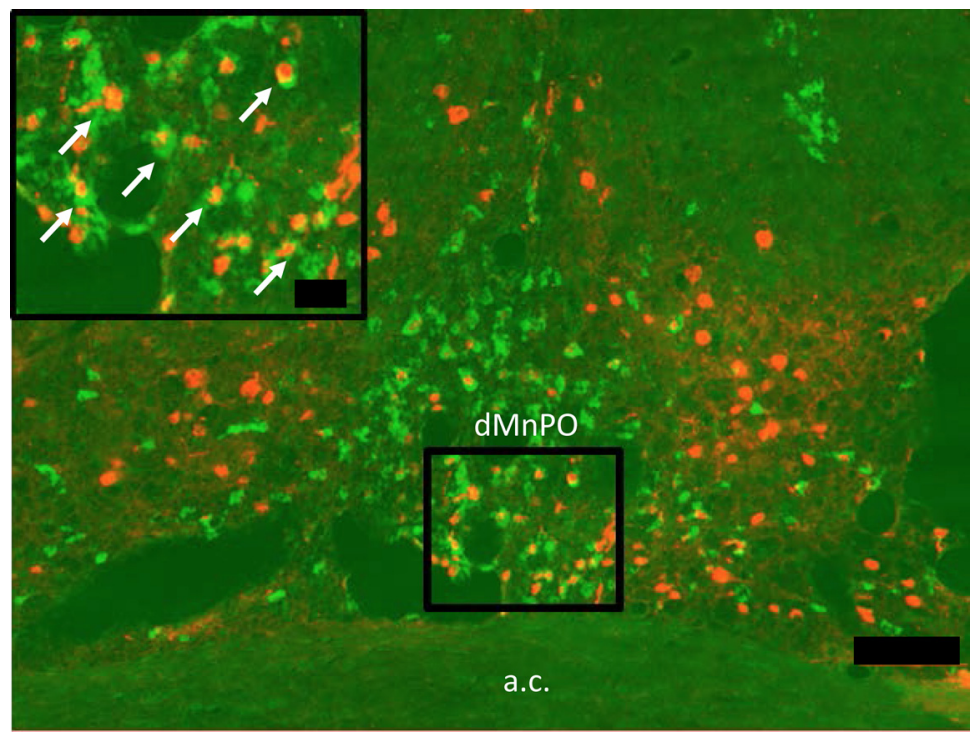

Figure 1. CaMKIla-positive MnPO neurons are glutamatergic. Representative image of the dorsal MnPO (dMnPO) with CaMKIlapositive MnPO neurons (red) and colocalization with vGLUT2 (green), as indicated in the inset by white arrows. Scale bar for inset, $25 \mu \mathrm{m}$. Main figure scale bar, $100 \mu \mathrm{m}$. Anterior commissure, a.c.

or control virus were stained for cFos (sc-253-G, goat polyclonal anti-Fos antibody, Santa Cruz Biotechnology, 1:1000). After $48 \mathrm{~h}$, sections were washed using PBS and transferred to a secondary antibody (BA-9500, biotinylated anti-goat, Vector Laboratories) for DAB reaction and labeling. After the DAB reaction, the sections were washed and placed in the primary antibody (ab167453, rabbit polyclonal anti-mCherry, Abcam 1:500) and incubated for an additional $48 \mathrm{~h}$ followed by incubation with a Cy3-conjugated anti-rabbit antibody (711-165-152, Jackson ImmunoResearch) for 4-5 h. The sections were then mounted on gelatin-coated slides, dried, and coverslipped with Permount for imaging. All antibodies were diluted to the final concentration with PBS diluent $(0.25 \%$ Triton X-100, 3\% horse serum, and $96.75 \%$ PBS).

Sections were examined using light microscopy to identify Fos-positive cells. Excitation wavelengths of 550$570 \mathrm{~nm}$ were used for emission of mCherry immunofluorescence. Images were captured using an epifluorescent microscope (Olympus BX41, Olympus) equipped with a digital camera (Olympus DP70) to image sections. Care was taken to ensure that sections included in this study were sampled from the same plane in each brain section. Colocalization was determined by quenching produced in cells with nuclear fos staining and cytosolic mCherry staining, as previously described (Grindstaff et al., 2000). Brightfield and fluorescent images were merged for analysis of the MnPO using ImageJ (NIH). Fos-positive neurons and their colocalization in the MnPO was determined blind to experimental conditions of the subjects.

\section{Statistics}

Electrophysiology data were tested for differences in baseline activity, changes in peak firing rate (defined as the 10-s bin with the lowest firing rate), and percentage change baseline firing rate using one-way ANOVA or twoway repeated measures ANOVA. Two-way repeated mea- sures ANOVA was used to compare changes in water consumption between the $G_{i}$ DREADD-injected group and control group and treatment (CNO vs VEH), followed by Tukey's post hoc test. Analyses of plasma vasopressin concentrations, plasma osmolality, and cell counts for neuronal phenotyping were figured using one-way ANOVA followed by Tukey's post hoc test. Tukey's post hoc analysis was used when performing multiple comparisons. Holm-Sidak post hoc test was used to perform comparisons to a determined control. Statistical significance is defined at an $\alpha$ level of 0.05 and exact $p$ values are reported. Values are reported as mean \pm SEM. All statistics were performed in SigmaPlot.v.12.0 (Systat Software).

\section{Results}

\section{CaMKIla neurons in the MnPO signal through excitatory pathways}

Experiments were conducted to determine the neurochemical phenotype of MnPO neurons that were targeted by the viral vectors with CaMKIla promoters used in this study. In situ hybridization for vGLUT2 was used to identify putative excitatory MnPO neurons ( $n=5$ rats, two to three sections per rat). An example of vGLUT2 in situ hybridization and CaMKIla immunohistochemistry in the dorsal $\mathrm{MnPO}$ is shown in Figure 1. The results show that $89.17 \pm 1.32 \%$ of CaMKIla neurons that express the viral construct co-localize with vGLUT2 message, indicating that the CaMKIla-expressing neurons in the MnPO are primarily glutamatergic and would likely be involved in excitatory signaling.

\section{Electrophysiology results}

CNO significantly attenuates basal firing rate in $G_{i}$ DREADD-expressing neurons

Intracellular recordings To verify that $\mathrm{CNO}$ was not having off-target effects, whole cell current clamp experiments 
A
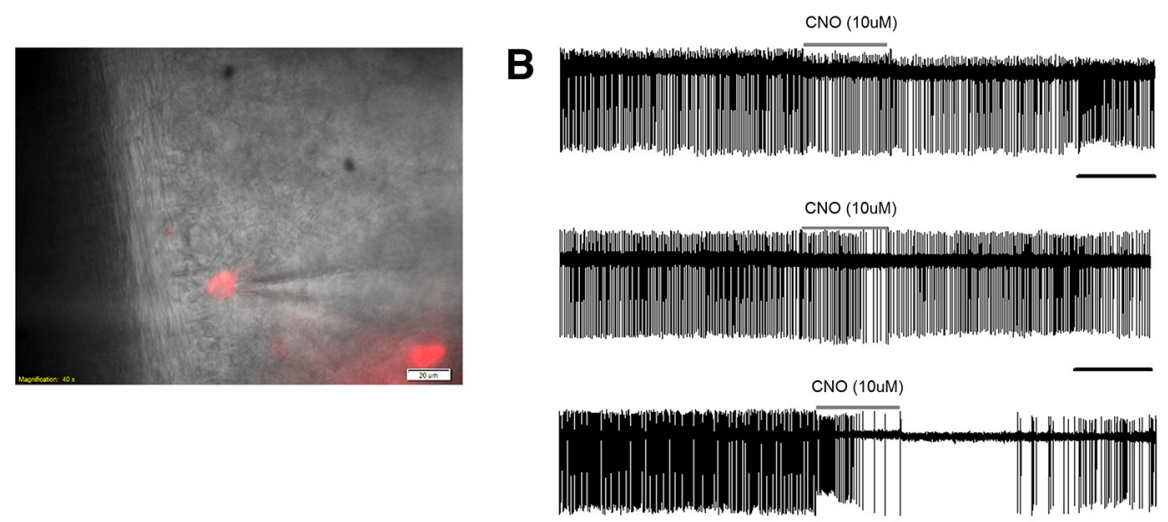

C

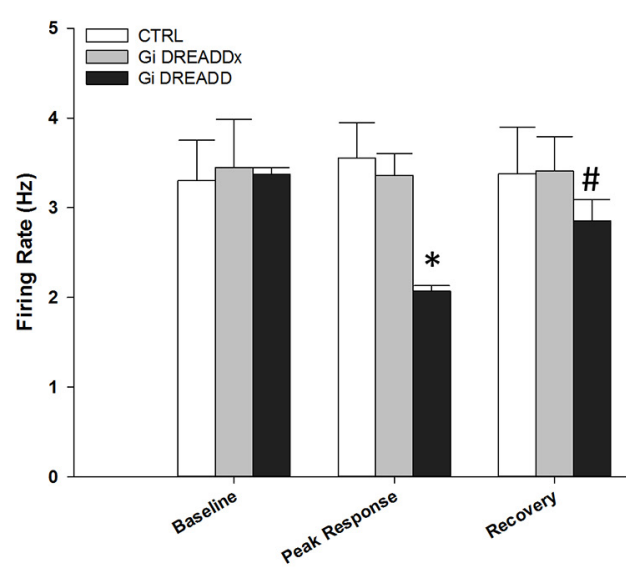

D

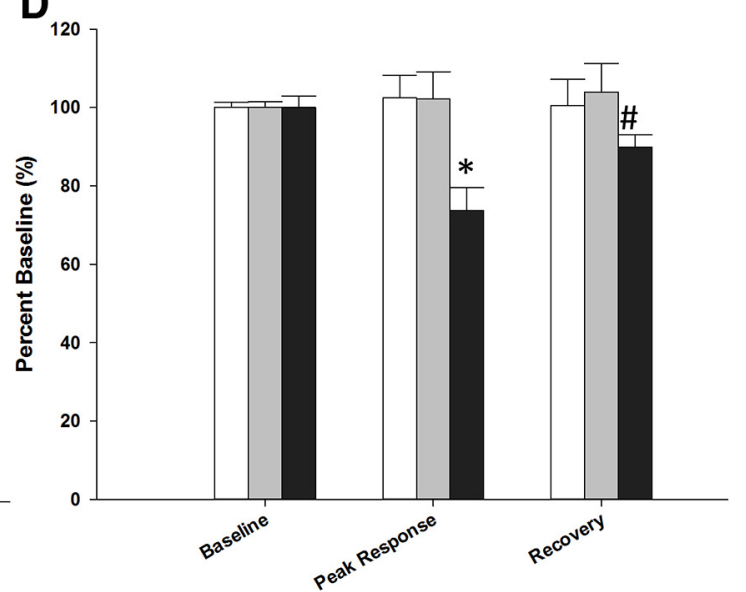

Figure 2. CNO-mediated inhibition significantly attenuates basal firing rate in $G_{i} D R E A D D-l a b e l e d$ neurons. $\boldsymbol{A}$, Representative image showing loose-cell patch recording of a $G_{i}$ DREADD-labeled ( $G_{i}$ DREADD) neuron (red). Scale bar, $20 \mu \mathrm{m}$. $\boldsymbol{B}$, Representative raw trace recordings of a control (CTRL) neuron (top, $n=13$ neurons, six rats), $G_{i}$ DREADD-unlabeled ( $G_{i}$ DREADDx) neuron (middle, $n=19$ neurons, six rats), and $G_{i}$ DREADD neuron (bottom, $n=17$ neurons, six rats). Scale bar, $10 \mathrm{~s}$. $C$, CNO-mediated inhibition significantly attenuated basal firing rate of $G_{i}$ DREADD neurons (peak response) compared to baseline and recovery, and compared to CTRL and $G_{i}$ DREADDx. $\boldsymbol{D}$, CNO-mediated inhibition significantly attenuated basal firing rate of $G_{i}$ DREADD neurons (peak response), represented as a percentage baseline, compared to $G_{i}$ DREADDx and CTRL neurons; $* p<0.050$, compared to peak response between groups; $\# p<0.050$, compared to baseline within group. Data are presented as mean and SEM.

(intracellular recordings) were conducted for more direct measurements on $\mathrm{G}_{\mathrm{i}}$ DREADD $(n=5$, three rats, two slices per rat) and $G_{i}$ DREADDx ( $n=7$, four rats, two slices per rat) neurons within the same section. As previously described (Grob et al., 2004), neurons were slightly depolarized with current injection to generate a regular spiking activity (range, -50 to $-40 \mathrm{mV}$ ) and allowed to stabilize in aCSF for $5 \mathrm{~min}$ before CNO exposure. There was a significant effect of treatment on neuron type $\left(F_{(3,20)}\right.$ $=21.175, p<0.001$, one-way ANOVA; data not shown). CNO exposure caused a significant membrane hyperpolarization in $G_{i}$ DREADD neurons between aCSF baseline and peak response $(-6.1 \pm 0.9 \mathrm{mV} ; p=0.001)$ but no significant change in membrane potential of the $G_{i} D R E-$ ADDx neurons in the presence of CNO compared to aCSF baseline $(2.1 \pm 0.9 \mathrm{mV} ; p=0.979)$. These results indicate that CNO and the DREADD-induced inhibition of the $G_{i}$ DREADD neurons did not have an effect on local circuitry.

Extracellular recordings Loose cell recordings were made from brain slices containing the MnPO two weeks after the rats were injected with AAVs containing either $G_{i}$
DREADD or the control (CTRL) construct. Cells expressing the constructs were easily identifiable by expression of the mCherry reported (Fig. $2 A$ ). There were no differences in the rates of spontaneous activity of MnPO neurons transfected with either virus $\left(G_{i}\right.$ DREADD, $3.37 \pm 0.1$ $\mathrm{Hz} ; n=17$, six rats, two slices per rat; CTRL, $3.30 \pm 0.5$ $\mathrm{Hz} ; n=13$, six rats, two slices per rat) or unlabeled cells in slices from rats injected in the MnPO with the $G_{i}$

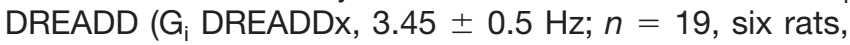
two slices per rat; $F_{(2,46)}=0.0306, p=0.970$, one-way ANOVA).

Focal CNO application $(10 \mu \mathrm{M})$ significantly decreased the spontaneous activity of the $G_{i}$ DREADD MnPO neurons $\left(F_{(1,32)}=16.22, p<0.001\right.$, one-way ANOVA; Fig. $\left.2 B\right)$. When calculated as a percentage change from baseline, only $G_{i} D R E A D D$ neurons showed a significant decrease in activity from baseline, or peak response (peak: defined as the 10-s bin with the lowest firing rate), that recovered after $172.4 \pm 37.8 \mathrm{~s}\left(F_{(2,46)}=10.050, p=0.001\right.$, one-way ANOVA; Tukey's post hoc, baseline vs peak $p=0.004<$ 0.001 and baseline vs recovery, $p=0.024$; Fig. $2 D$ ). There 
was also a significant difference in peak response to CNO in the $\mathrm{G}_{\mathrm{i}}$ DREADD neuron firing rate $\left(F_{(2,46)}=10.832, p<\right.$ 0.005 , one-way ANOVA) compared to CTRL $(p<0.001)$ and $G_{i}$ DREADDx neurons $(p<0.001)$ using Holm-Sidak post hoc test.

Analysis of the firing rates $(\mathrm{Hz})$ of neurons from each group produced similar results (Fig. 2C). Focal CNO application did not significantly influence the spontaneous activity of the $\mathrm{G}_{\mathrm{i}}$ DREADDx $\left(F_{(2,36)}=0.0808, p=0.923\right.$, one-way ANOVA) or CTRL neurons $\left(F_{(2,54)}=0.0119, p=\right.$ 0.988 , one-way ANOVA; Fig. 2D). Additional experiments were therefore conducted to determine whether CNO could block changes in activity in CaMKIla MnPO neurons produced by either ANG II or HTN.

\section{CNO blocks ANG II-induced excitation in $\mathrm{G}_{\mathrm{i}}$ DREADD-expressing neurons}

Next, the effects of CNO on ANG II evoked responses in $G_{i}$ DREADD MnPO neurons were tested. In these experiments, focally applied ANG II (10 $\mu \mathrm{M})$ was administered during bath applications of CNO $(500 \mathrm{nM})$. Bath application of CNO containing aCSF significantly reduced the spontaneous activity of $\mathrm{G}_{\mathrm{i}}$ DREADD neurons $(n=15$, six rats, two slices per rat) as compared to aCSF alone $(n=$ 7 , three rats, two slices per rat). In contrast bath application of CNO did not affect the activity of $G_{i}$ DREADDx neurons from the same slices $(n=15$, six rats, two slices per rat). In the $G_{i} D R E A D D-p o s i t i v e$ neurons, this inhibition of spontaneous activity by CNO bath application was significant at $170 \mathrm{~s}$ after the start of CNO exposure and was maintained throughout the duration of the protocol $\left(F_{(2,39)}=5.577, p=0.007\right.$, two-way repeated measures ANOVA; Fig. $3 A, B)$. When analyzed as a percentage change from aCSF baseline, the activity of $G_{i}$ DREADD cells was significantly reduced to $20 \%$ of baseline during CNO bath application, whereas $G_{i}$ DREADDx cells were not affected $\left(F_{(58,1131)}=4.221, p<0.001\right.$, two-way repeated measures ANOVA; Fig. $3 C, D)$.

During aCSF bath application, focally applied ANG ॥ significantly increased the firing rate of seven out of seven $\mathrm{G}_{\mathrm{i}}$ DREADD neurons ( $n=7$, three rats, two slices per rat). The activity of $G_{i}$ DREADDx neurons was increased by focal ANG II during CNO bath application $\left(F_{(3,66)}=\right.$ $840.408, p<0.001$, one-way ANOVA). However, CNO bath application blocked the responses of 10 out of $15 \mathrm{G}_{\mathrm{i}}$ DREADD-labeled neurons to focally applied ANG II $p=$ 0.258 , Tukey's post hoc test; Fig. 3D). There was no significant difference in baseline firing rates between $G_{i}$ DREADD neurons before $C N O$ exposure or $G_{i}$ DREADDx neurons during $\mathrm{CNO}$ or aCSF exposure $\left(F_{(3,51)}=2.253\right.$, $p=0.093$, one-way ANOVA).

\section{CNO significantly attenuates excitation produced by hypertonic saline in $G_{i}$ DREADD-expressing neurons}

The effects of DREADD mediated inhibition on responses produced by bath application of HTN-aCSF (330 mOsm) was tested using MnPO neurons transfected with $G_{i}$ DREADD or unlabeled cells in the same brain slices $\left(G_{i}\right.$ DREADDx). The activity of seven out of seven $G_{i}$ DREADD neurons was significantly increased by HTN bath solution. As observed in the previous experiments, bath application of CNO decreased the basal activity of cells transfected with the $G_{i}$ DREADD construct but did not affect the activity of $G_{i}$ DREADDx cells (Fig. $4 A, B$ ).

Bath application of CNO did not influence the spontaneous activity or prevent increases in firing rate produced by HTN-aCSF in $\mathrm{G}_{\mathrm{i}}$ DREADDx neurons compared to aCSF baseline (Fig. 4A). In contrast, CNO decreased spontaneous activity in $G_{i}$ DREADD cells as compared to activity in normal aCSF $\left(F_{(2,41)}=112.004, p<0.001\right.$, Holm-Sidak post hoc analysis; Fig. $4 A$ ). $G_{i}$ DREADD neurons did not show a significant increase in firing rate associated with HTN-aCSF in the presence of CNO (Fig. 4C). Although the $G_{i}$ DREADD cells appeared to be more active during bath application of $\mathrm{CNO}$ and $\mathrm{HTN}-\mathrm{aCSF}$, their average firing frequency was not different from CNO alone (Fig. 4C). The changes in firing rate of $\mathrm{MnPO}$ neurons was influenced by both the treatment and time as indicated by a statistically significant interaction $\left(F_{(89,2609)}=2.712, p<0.001\right.$, twoway repeated measures ANOVA).

CNO bath application significantly attenuated changes in activity expressed as percentage of baseline activity in $G_{i}$ DREADD neurons ( $n=15$, six rats, two slices per rat, $p$ $<0.001$, Tukey's post hoc analysis). This was not the case for $G_{i}$ DREADDx neurons ( $n=14$, six rats, two slices per rat) compared to aCSF bath applied $G_{i}$ DREADD neurons $(n=7$, two rats, two slices per rat; Fig. $4 A, B)$. $\mathrm{HTN}-a C S F$ significantly increased the firing rate of unlabeled cells compared to baseline and aCSF recordings $\left(F_{(3,52)}=7.119, p<0.001\right.$, one-way ANOVA; Fig. $\left.4 C\right)$. $\mathrm{G}_{\mathrm{i}}$ DREADDx neurons had significantly increased firing rates when exposed to HTN-aCSF with or without CNO $(p<$ 0.01 , Tukey's post hoc analysis; Fig. 4C). When the bath solution was changed to normal aCSF from HTN-aCSF, the activity of $G_{i} D R E A D D x$ cells significantly decreased to a rate that was no different from $\mathrm{CNO}$ and normal aCSF $\left(p=0.301\right.$ baseline compared to aCSF; Fig. $4 C$ ). When $\mathrm{G}_{i}$ DREADD were exposed to HTN-aCSF without CNO, their firing rate significantly increased as compared to the aCSF baseline $(p<0.001$ for all time points, Tukey's post hoc analysis; Fig. $4 C$ ) and compared to CNO ( $p<0.001$ for all time points, Tukey's post hoc analysis), but not when compared to $G_{i}$ DREADDx neurons exposed to CNO.

In $G_{i}$ DREADD neurons, there was a significant decrease in firing rate during $\mathrm{CNO}$ and $\mathrm{HTN}$ exposure compared to aCSF bath application $\left(F_{(3,56)}=30.084 ; p<\right.$ 0.001 , one-way ANOVA; $p=0.001$, Tukey's post hoc analysis). Although there was a trend for increased activity in $G_{i}$ DREADD neurons exposed to HTN-aCSF after CNO was washed out (Fig. 4C), this change was not significantly different from CNO with HTN-aCSF $(p=0.917$, Tukey's post hoc analysis).

\section{Drinking responses}

Based on the results of the electrophysiological studies, we tested the effects of intraperitoneal CNO $(10 \mathrm{mg} / \mathrm{kg})$ on drinking responses produced by ANG II $(2 \mathrm{mg} / \mathrm{kg}$, s.c.) in one group of rats and $3 \%$ HTN ( $1 \mathrm{ml} / 100-\mathrm{g} \mathrm{bw}$, s.c.) in a separate group of rats. Control experiments using equal volumes of $0.9 \%$ saline (SAL) tests were also conducted for each group. 

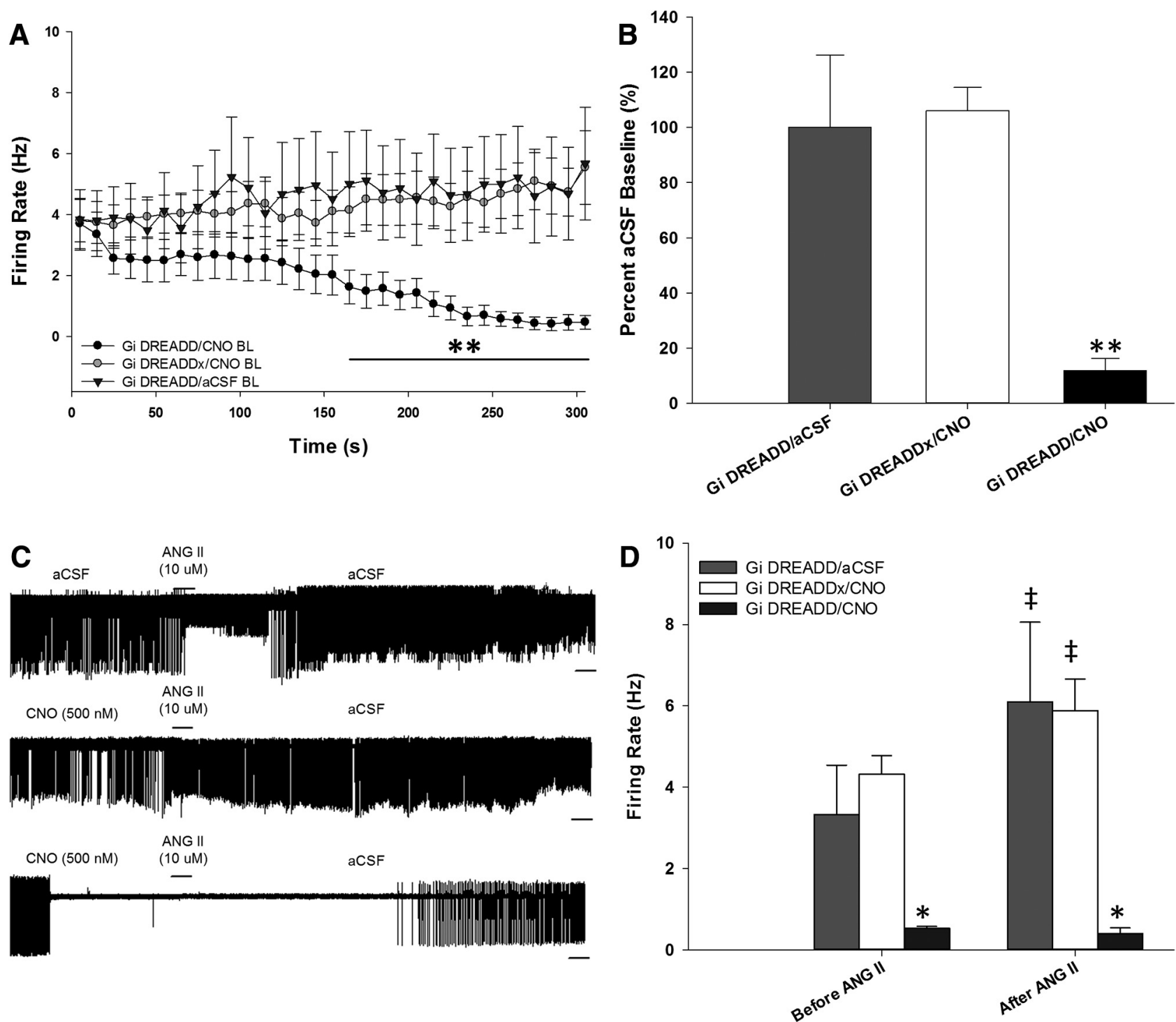

Figure 3. CNO-mediated inhibition blocks ANG II-induced excitation. $\boldsymbol{A}$, CNO bath application significantly inhibits basal firing rate of $\mathrm{G}_{\mathrm{i}}$ DREADD neurons ( $n=15$ neurons, six rats), but does not affect spontaneous activity in control ( $n=7$ neurons, three rats) or $\mathrm{G}_{\mathrm{i}}$ DREADD-unlabeled ( $\mathrm{G}_{\mathrm{i}}$ DREADDx) neurons ( $n=15$ neurons, six rats); **p $<0.050$ compared to each group. $\boldsymbol{B}$, $\mathrm{G}_{\mathrm{i}} \mathrm{DREADD}$ neurons during $\mathrm{CNO}$ bath application reduced firing rate to $20 \%$ of percentage aCSF baseline but did not affect baseline of $\mathrm{G}_{\text {i }}$ DREADDx neurons. $\boldsymbol{C}$, Representative raw trace recordings of a $G_{i}$ DREADD neuron during aCSF (control conditions) exposure (top), $G_{i}$ DREADDx neuron (middle), and $G_{i}$ DREADD neuron (bottom) with the two latter exposed to CNO during baseline. Scale bar, $10 \mathrm{~s}$. $D$, Focal ANG II application significantly increased firing rate of $G_{i}$ DREADDx neurons during CNO exposure and $G_{i}$ DREADD neurons during aCSF exposure; $\neq p<0.001$. CNO-mediated inhibition blocked ANG II excitation of $G_{i}$ DREADD neurons and displayed significantly reduced firing rate compared to $\mathrm{G}_{i}$ DREADDx neurons; $* p<0.001$. Data are presented as mean and SEM.

\section{Acute CNO-induced inhibition significantly attenuates ANG II-induced thirst responses}

When rats were pretreated with CNO or VEH injections followed by ANG II treatment, there was an associated increase in water consumption by rats during the 3-h time period in both the $\mathrm{G}_{\mathrm{i}}$ DREADD rats $(n=10)$ and CTRL rats $(n=10)$. Two-way repeated measures ANOVA analysis indicated a significant $A A V$ variant dependent difference in water consumption $\left(F_{(1,18)}=18.925, p<0.001\right.$, twoway repeated measures ANOVA; Fig. $5 A$ ) and treatment $\left(\mathrm{VEH}\right.$ or CNO) received $\left(F_{(5,86)}=69.735, p<0.001\right.$, two-way repeated measures ANOVA). There was no sig- nificant difference between groups during the $0.9 \% \mathrm{SAL}$ tests [first treatment $p=0.989$, last treatment $p=0.935$, first treatment vs last treatment $p=0.999$ (CTRL) and $p=$ $0.998\left(G_{i}\right.$ DREADD)] or during VEH and ANG II test $p=$ 0.074 ) in the first treatment. During the CNO and ANG II test, however, the $G_{i}$ DREADD group had approximately a $50 \%$ reduction in water consumed over the 3 -h period compared to the CTRL group ( $p<0.001$, first treatment). This was also the case compared to the VEH and ANG II exposure ( $p=0.005$, first treatment). Independent of VEH or CNO treatment, ANG II still resulted in significantly elevated drinking response compared to physiologic sa- 
A
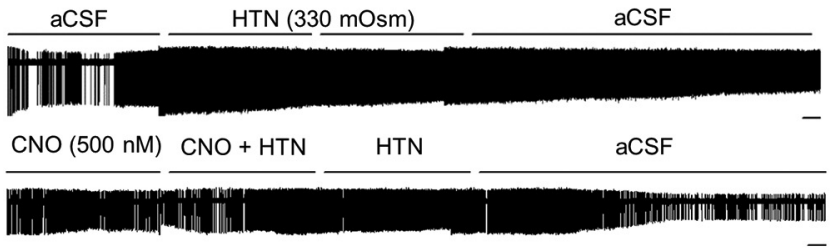

CNO $(500 \mathrm{nM})$ CNO + HTN

||
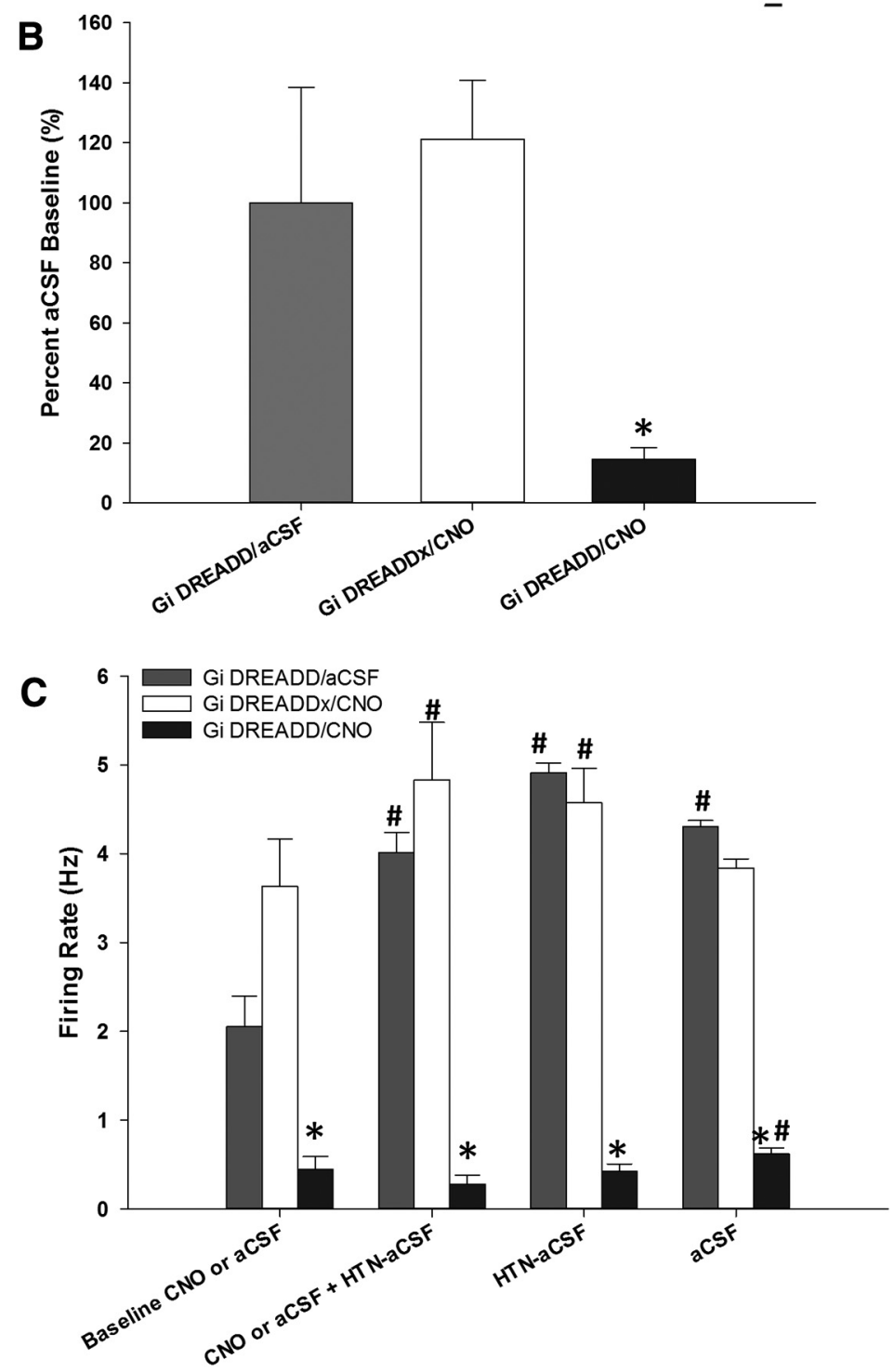

Figure 4. CNO-mediated inhibition attenuates firing rate during hyperosmotic challenges. $\boldsymbol{A}$, Representative raw trace recordings of a $\mathrm{G}_{\mathrm{i}}$ DREADD neuron in the absence of $C N O$, using aCSF for control conditions (top), $G_{i}$ DREADD-unlabeled ( $G_{i}$ DREADDx) neuron (middle), and $G_{i}$ DREADD neuron (bottom), both latter neurons exposed to CNO. Scale bar, 10 s. Hypertonic aCSF, HTN-aCSF. B, CNO significantly inhibits basal firing rate compared to percentage aCSF baseline of $G_{i} D R E A D D$ neurons. $\boldsymbol{C}$, Hyperosmotic challenges significantly increased firing frequency of $G_{i}$ DREADD neurons during aCSF exposure and $G_{i}$ DREADDx neurons during $C N O$ exposure (unaffected). $C N O$ significantly attenuated firing rate of $G_{i}$ DREADD neurons compared to $G_{i}$ DREADDx neurons. There was an observed increase in firing rate in the absence of $C N O$ during HTN-aCSF bath application, which became significant during aCSF bath application; $* p<0.001$ compared to $G_{i}$ DREADDx neurons exposed to $C N O$ and $G_{i}$ DREADD neurons during aCSF exposure; $\# p<0.05$ compared to time points within group. Data are presented as mean and SEM. 

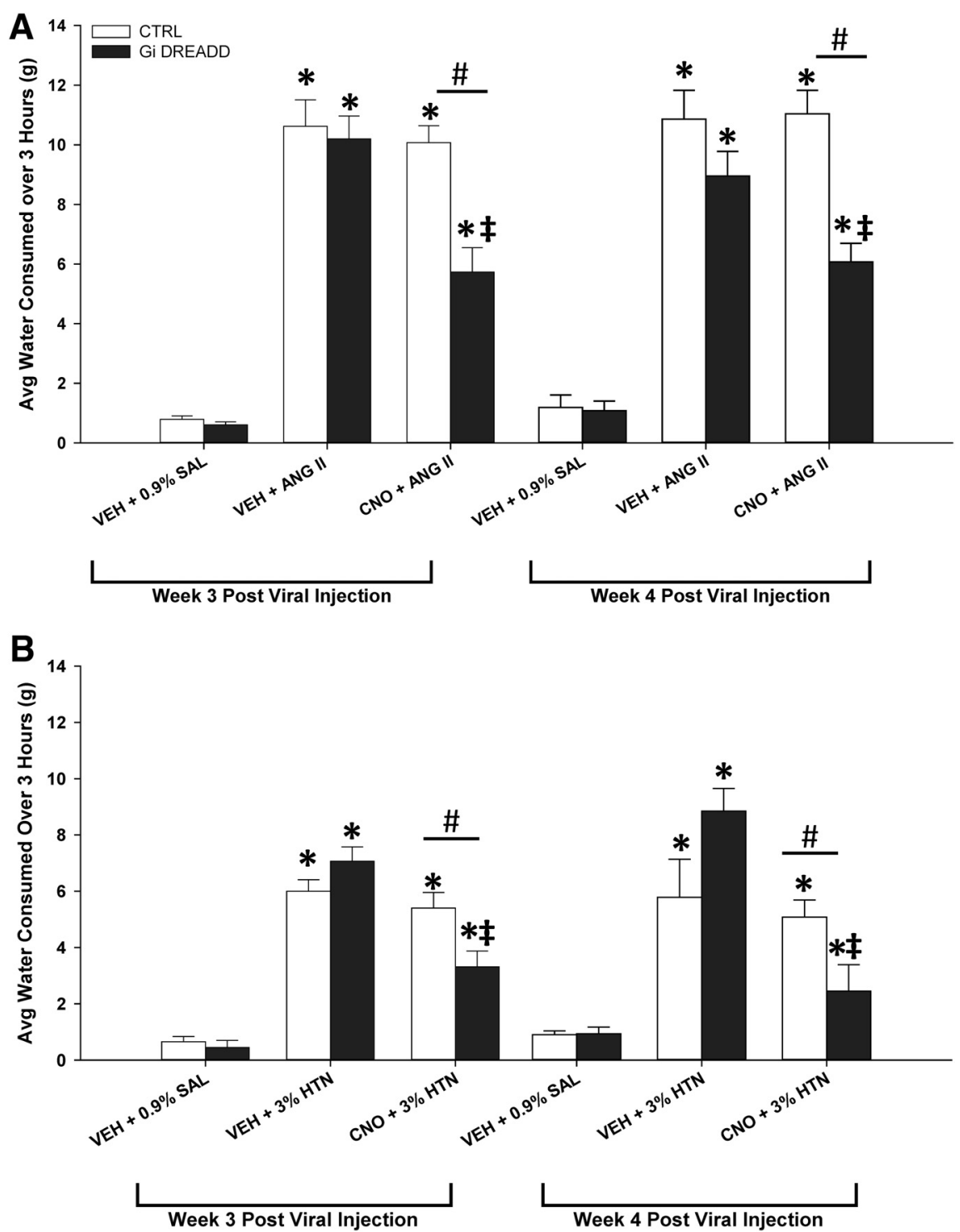

Figure 5. Acute MnPO inhibition attenuates evoked drinking responses. A, ANG II significantly increased water consumption compared to volume control tests (VEH $+0.9 \% \mathrm{SAL}$ ) in both $\mathrm{G}_{\mathrm{i}}$ DREADD and CTRL (VEH + ANG II) rats $(n=10$ rats). CNO-mediated inhibition significantly attenuated this increase in $\mathrm{G}_{\mathrm{i}}$ DREADD (CNO + ANG II) rats $(n=10$ rats). B, Hypertonic saline (3\% HTN) significantly increased water consumption compared to VEH + 0.9\% SAL tests in both $\mathrm{G}_{\mathrm{i}}$ DREADD and CTRL (VEH + 3\% HTN) rats ( $n=6$ rats) and CNO-mediated inhibition significantly attenuated this increase in $\mathrm{G}_{\mathrm{i}}$ DREADD rats $(n=6$ rats); $* p<0.005$ compared to VEH $+0.9 \%$ SAL in respective group $(\boldsymbol{A}$ or $\boldsymbol{B})$; $\neq p=<0.050$ compared to VEH and ANG II $(\boldsymbol{A})$ and $3 \%$ HTN $(\boldsymbol{B})$ exposure in respective group; \#p $<0.015$ compared to CTRL + CNO + ANG II $(\boldsymbol{A})$ and CTRL + CNO + 3\% HTN $(\boldsymbol{B})$. Data are presented as mean and SEM.

line volume control studies (all groups, $p<0.001$, Tukey's post hoc analysis).

One week later, ANG II significantly increased drinking behavior in the same manner compared to $0.9 \%$ SAL tests (all groups $p<0.001$, Tukey's post hoc analysis). There was no significant difference in thirst response between $G_{i}$ DREADD or CTRL groups during VEH and ANG II exposure ( $p=0.292$, last treatment). During the CNO and ANG II treatment, however, the $G_{i}$ DREADD group had approximately a $50 \%$ reduction in water consumed over the 3-h period compared to the CTRL group, similar to what was observed in the first treatment $(p<$ 0.001 , last treatment).

\section{Acute CNO-induced inhibition significantly} attenuates hypertonic saline-induced thirst

A separate cohort of rats were injected in the MnPO with either $\mathrm{G}_{\mathrm{i}}$ DREADD ( $n=6$ rats) or CTRL ( $n=6$ rats) virus and used to test whether or not CNO pretreatment would block the effects of $3 \%$ HTN injections on drinking behavior. As in the ANG II drinking studies, there was a significant interaction between AAV variant, pretreatment and $3 \%$ HTN exposure $\left(F_{(5,71)}=3.317, p=0.015\right.$, twoway repeated measures ANOVA; Fig. 5B). When either of the groups were pretreated with VEH followed by $3 \%$ HTN, rats drank a significant amount of water over the 3-h time period compared to $0.9 \%$ SAL tests (Tukey's post 

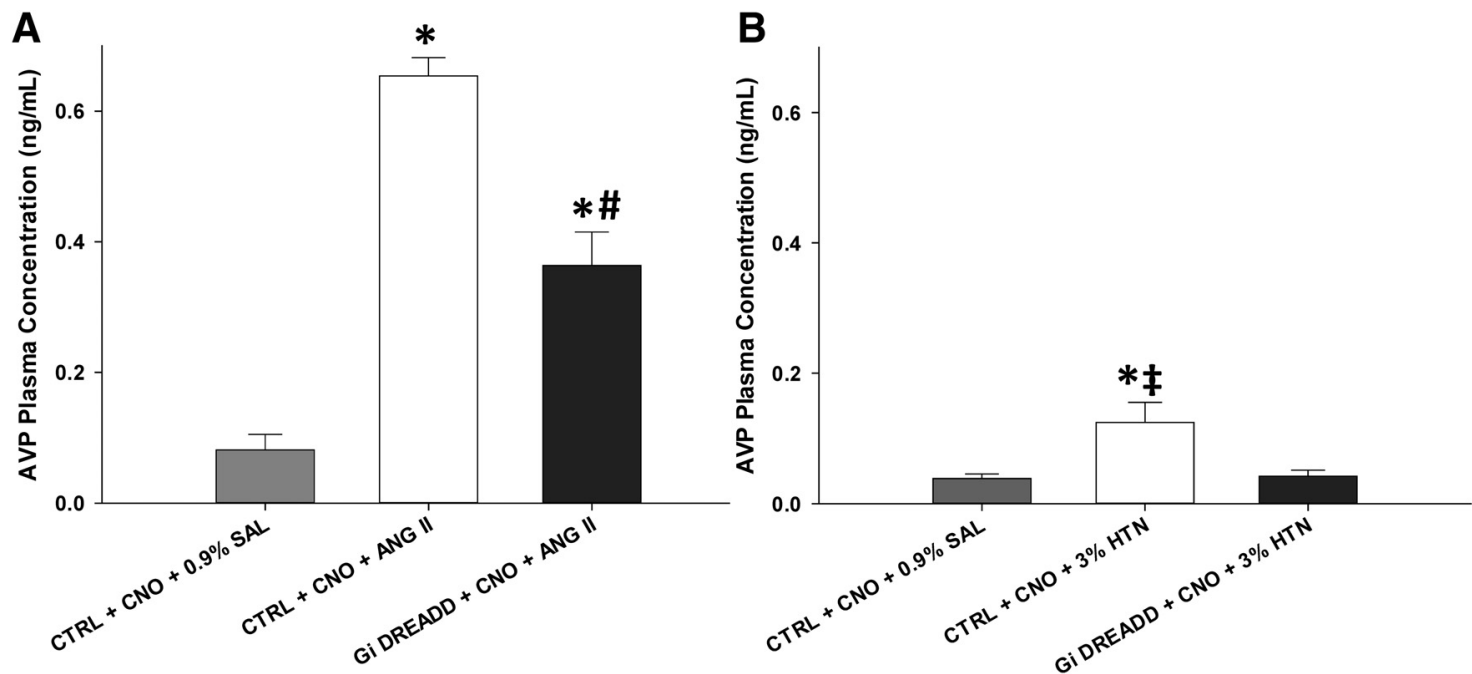

Figure 6. CNO-induced inhibition of CaMKIla MnPO neurons significantly attenuates evoked increases of plasma AVP. A, ANG II significantly increased plasma AVP concentration in CTRL rats (CTRL + CNO + ANG II), but this increase was significantly attenuated during CNO-mediated inhibition in $\mathrm{G}_{\mathrm{i}}$ DREADD ( $\mathrm{G}_{\mathrm{i}}$ DREADD + CNO + ANG II) rats. B , Hypertonic saline (3\% HTN) significantly

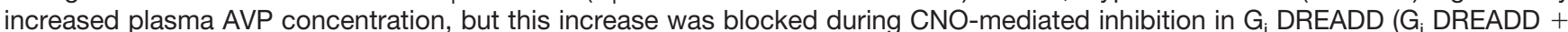
$\mathrm{CNO}+3 \% \mathrm{HTN}$ ) rats; $* p<0.02$ compared to respective controls (CTRL + CNO + 0.9\% SAL); $\neq p<0.050$ compared to VEH and $3 \%$ HTN exposure; \#p $<0.001$ compared to CTRL + CNO + ANG II. Data are presented as mean and SEM.

hoc analysis, $0.9 \%$ SAL vs CTRL + VEH + 3\% HTN $p<$ $0.001,0.9 \%$ SAL vs $G_{i}$ DREADD + VEH + 3\% HTN $p<$ $0.001, \mathrm{CTRL}+\mathrm{VEH}+3 \% \mathrm{HTN}$ vs $\mathrm{G}_{\mathrm{i}}$ DREADD + VEH + $3 \%$ HTN group $p=0.794$; Fig. $5 B$ ). However, when groups were pretreated with CNO before $3 \%$ HTN, the drinking response of the rats injected with $G_{i}$ DREADD were significantly attenuated as compared to their responses to VEH and 3\% HTN treatment (Tukey's post hoc analysis; $0.9 \%$ SAL vs CTRL + CNO + 3\% HTN $p<$ $0.001,0.9 \%$ SAL vs DREADD + CNO + 3\% HTN $p<$ $0.001, \mathrm{CTRL}+\mathrm{CNO}+3 \%$ HTN vs DREADD $+\mathrm{CNO}+$ $3 \%$ HTN $p=0.011$; Fig. $5 B$ ). Rats were injected with the same volume of $0.9 \% \mathrm{SAL}$ and this treatment did not significantly affect water intake between groups $(p=$ 0.799 , Tukey's post hoc analysis; Fig. $5 B$ ).

After one week, the same rats were retested. Injections of HTN significantly increased water intake compared to $0.9 \%$ SAL tests (all groups, $p<0.001$, Tukey's post hoc analysis). There was no significant difference in water intake between groups during VEH and 3\% HTN exposure ( $p=0.319$, last treatment). During the CNO and $3 \%$ HTN treatment, the $G_{i}$ DREADD group had approximately a $50 \%$ reduction in water consumed over the 3 -h period compared to the CTRL group, similar to what was observed during the first treatment ( $p=0.028$, last treatment).

\section{Arginine vasopressin responses}

Two days after completing the last drinking tests, rats were injected with $\mathrm{VEH}$ or $\mathrm{CNO}(10 \mathrm{mg} / \mathrm{kg}$, i.p.) followed by either ANG II (2 mg/kg, s.c.), 3\% HTN (1 ml/100-g bw, s.c.) or $0.9 \%$ SAL (volume control) 30 min later, as described in the drinking tests. Blood samples were taken and analyzed for plasma AVP concentrations.

\section{CNO-induced inhibition significantly attenuates ANG II-induced plasma vasopressin release}

The effects of DREADD inhibition in the MnPO on ANG II-induced AVP release was tested. Rats injected in the $\mathrm{MnPO}$ with the control vector (CTRL) that were pretreated with CNO and administered ANG II (CTRL + CNO + ANG II) had significantly increased plasma $\operatorname{AVP}\left(F_{(2,23)}=\right.$ 44.963, $p<0.001$, one-way ANOVA; Fig. $6 A)$. This increase in plasma AVP associated with ANG II treatment was significantly decreased by CNO-induced inhibition in the $G_{i}$ DREADD $\left(G_{i}\right.$ DREADD + CNO + ANG II) group $(p<$ 0.001 , Tukey's post hoc analysis). However, the changes in plasma AVP observed in the $G_{i}$ DREADD + CNO + ANG II were significantly increased compared to the vehicle control group (CTRL + CNO + 0.9\% SAL) suggesting the ANG II-induced AVP release was significantly attenuated, but not reduced to control levels by MnPO inhibition $(p<0.001$; Fig. $6 A)$.

\section{CNO-induced inhibition blocks hypertonic saline- induced plasma vasopressin release}

Plasma AVP concentration was significantly different between groups treated with $3 \%$ HTN compared to $0.9 \%$ $\operatorname{SAL}\left(F_{(2,15)}=6.443, p=0.010\right.$, one-way ANOVA; Fig. $\left.6 B\right)$, as well. Subcutaneous injection of $3 \%$ HTN significantly increased plasma AVP levels in the CTRL + CNO + 3\% HTN from the vehicle controls (CTRL + CNO + 0.9\% SAL) group ( $p=0.016$; Tukey's post hoc analysis). Consistent with the ANG II plasma AVP results, CNO pretreatment significantly attenuated this increase in the $G_{i}$ DREADD + CNO $+3 \%$ HTN group $(p=0.008$, Tukey's post hoc analysis). There was no significant difference in plasma AVP concentrations between the CTRL + CNO + 0.9\% SAL and the $\mathrm{G}_{i}$ DREADD + CNO + 3\% HTN group $p=$ 0.893 , Tukey's post hoc analysis). 

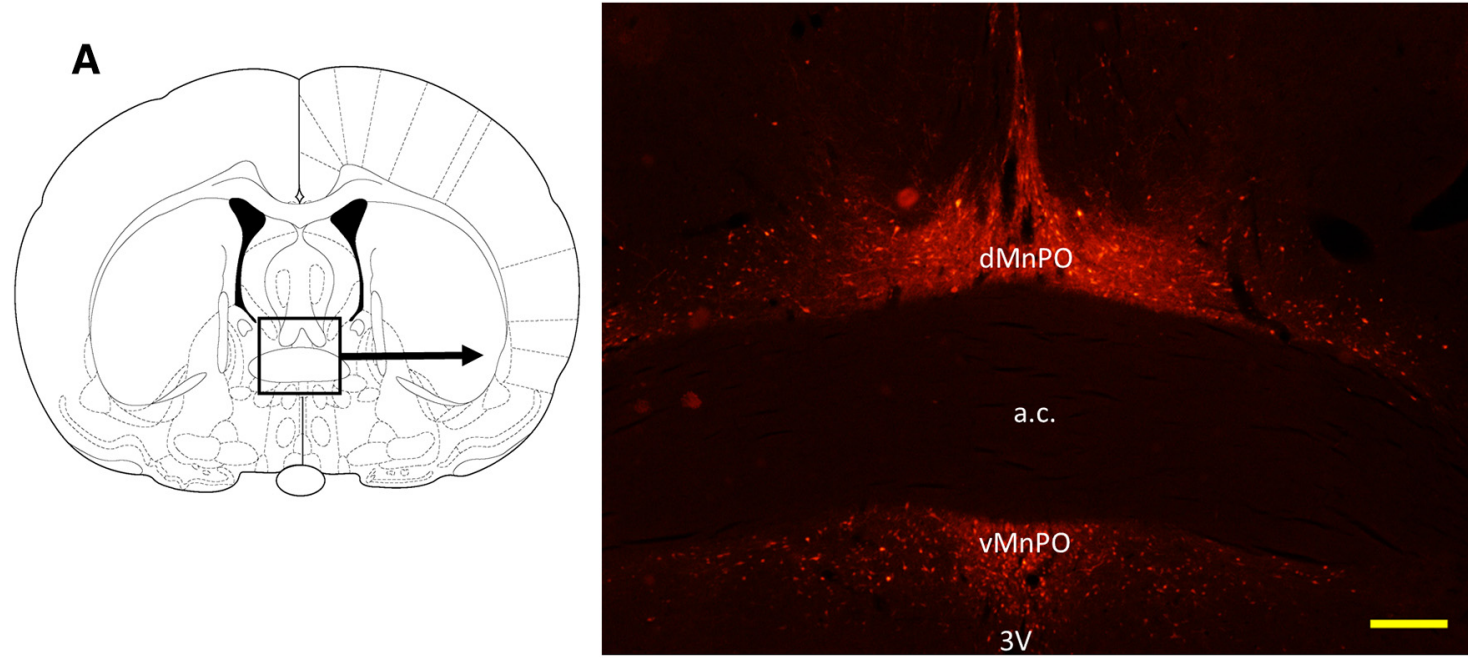

B $\quad C T R L+V E H+V E H$

$C T R L+C N O+V E H$

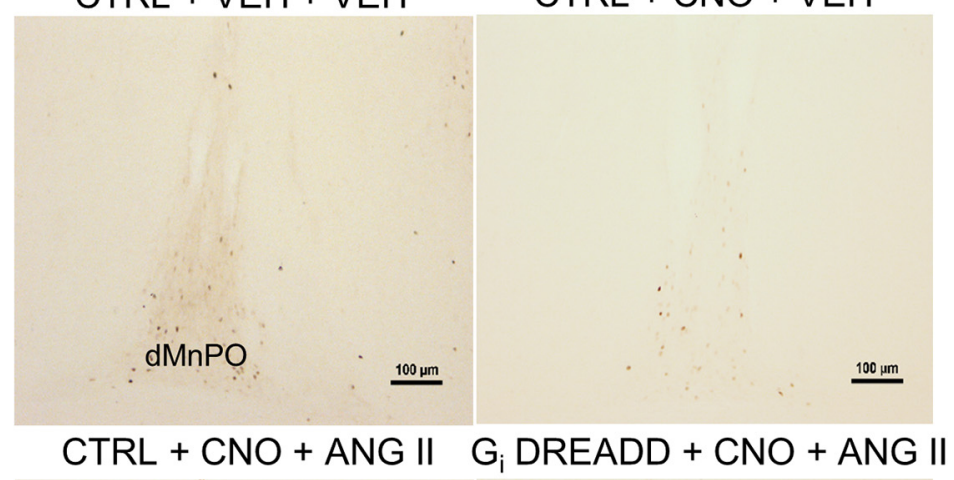

$100 \mu \mathrm{m}$

$100 \mu \mathrm{m}$

Figure 7. ANG II significantly increases Fos expression in the MnPO in control virus-injected rats, but is blocked during CNO-mediated inhibition in $\mathrm{G}_{\mathrm{i}} \mathrm{DREADD}$-injected rats. $\boldsymbol{A}$, Diagram showing representative mCherry labeling from AAV transfection in the MnPO. Scale bar, $250 \mu \mathrm{m}$. Third ventricle, 3V; anterior commissure, a.c.; dorsal MnPO, dMnPO; ventral MnPO, vMnPO. B, Representative Fos staining in the dMnPO of control virus-injected (CTRL) rats treated with CNO vehicle (VEH) and ANG II vehicle ( $0.9 \%$ SAL; $n=4$, two to four sections per rat; upper left panel), CTRL rats treated with $\mathrm{CNO}$ and $0.9 \% \mathrm{SAL}(n=4$, two to four sections per rat; upper right panel), CTRL rats treated with $\mathrm{CNO}$ and ANG II $(n=10$, two to four sections per rat; lower left panel), and $\mathrm{G}_{\mathrm{i}}$ DREADD-injected rats treated with CNO and ANG II $(n=10$, two to four sections per rat; lower right panel). Scale bar, $100 \mu \mathrm{m}$.

\section{Functional neuroanatomy}

Brains harvested from perfusions were analyzed for Fos and mCherry expression associated with the AAVs MnPO injections were verified by detecting the presence of mCherry immunofluorescence (Fig. 7A). Rats included in the study had mCherry expression isolated to the MnPO. Rats with injections or mCherry labeling outside of the $\mathrm{MnPO}$ were excluded from the study.

\section{CNO-induced inhibition during ANG II exposure significantly blocks CaMKIla+ Fos expression in the MnPO}

In rats treated with the control vector injections of $\mathrm{CNO}$ followed by subcutaneous injection of $0.9 \%$ saline (CTRL $+\mathrm{CNO}+0.9 \% \mathrm{SAL}$ ) produced Fos staining in the MnPO that was not different from Fos staining associated with VEH injections paired with $0.9 \%$ saline subcutaneously 
Table 1. Colocalization of ANG II-induced Fos expression with CaMKIla neurons in the MnPO

\begin{tabular}{|c|c|c|c|c|}
\hline Treatment & Total cFos + & Total CaMKIla+ & Total DL cells & $\%$ DL cFos + \\
\hline $\begin{array}{l}\text { Control } \\
\text { CNO + } 0.9 \% \text { SAL }\end{array}$ & $21.0 \pm 10.0$ & $182.8 \pm 54.8$ & $8.8 \pm 1.25$ & $50.2 \pm 17.9 \%$ \\
\hline $\begin{array}{l}\text { Control } \\
\text { VEH + } 0.9 \% \text { SAL }\end{array}$ & $25.8 \pm 7.8$ & $181.5 \pm 37.5$ & $15.1 \pm 3.4$ & $60.0 \pm 4.8 \%$ \\
\hline $\begin{array}{l}\text { Control } \\
\text { CNO + ANG II }\end{array}$ & $79.8 \pm 8.7 * *$ & $195.0 \pm 57.6$ & $44.0 \pm 7.8 * *$ & $54.4 \pm 6.7 \%$ \\
\hline $\mathrm{G}_{\mathrm{i}}$ DREADD CNO + ANG II & $41.9 \pm 7.2$ & $206.5 \pm 41.0$ & $2.9 \pm 1.0$ & $6.5 \pm 2.9 \% * *$ \\
\hline
\end{tabular}

ANG II exposure significantly increases Fos expression in the MnPO, specifically in CaMKIla-positive neurons. CNO-mediated inhibition significantly attenuated ANG II-induced Fos expression in the MnPO in $\mathrm{G}_{\mathrm{i}}$ DREADD-injected rats, with inhibition of the CaMKIla neuronal phenotype; Bold text and $* * p<0.001$ compared to all groups. Data are presented as mean \pm SEM.

(CTRL + VEH + 0.9\% SAL; Fig. 7B; Table 1). Rats treated with the control vector and injected with CNO and ANG II $(C T R L+C N O+$ ANG II) had a significant increase in Fos staining in the $\mathrm{MnPO}\left(F_{(3,24)}=32.575, p<0.001\right.$, one-way ANOVA; Fig. 7B; Table 1).

In the $\mathrm{G}_{i}$ DREADD + CNO + ANG II group $(n=10$, two to four MnPO sections per rat), the increase in Fos staining in the MnPO was significantly decreased as compared to the CTRL + CNO + ANG II group $(n=10$, two to four MnPO sections per rat; Tukey's post hoc analysis, $p<$ 0.001 ) but not different from the Fos staining observed in $\mathrm{CTRL}+\mathrm{VEH}+0.9 \%$ SAL $(p=0.170)$ or CTRL + CNO + $0.9 \% \operatorname{SAL}(p=0.531)$ rats. Fos staining in the MnPO was significantly increased in CTRL + CNO + ANG II rats compared to either the CTRL + VEH $+0.9 \%$ SAL $(p<$ $0.001)$ or CTRL + CNO + VEH $(p<0.001)$ rats. Elevated $\mathrm{MnPO}$ Fos expression was blunted in $\mathrm{G}_{\mathrm{i}}$ DREADD + CNO + ANG II rats $(p<0.001)$. There was no significant difference between CTRL groups treated with either VEH or CNO $(p=0.916)$ and $0.9 \%$ SAL.

\section{CaMKIla neuron inhibition in the MnPO significantly decreases ANG II-evoked effects in downstream nuclei}

As expected, ANG II significantly increased Fos staining in $\mathrm{CaMKIIa}^{+}$neurons $\left(F_{(3,12)}=17.430, p<0.001\right.$, oneway ANOVA). The numbers of Fos and CaMKIla labeled in MnPO were significantly higher in the CTRL + CNO + ANG II group compared to the CTRL + VEH + 0.9\% SAL $(n=4$, two to four MnPO sections per rat; $p<0.001)$ and CTRL + CNO + $0.9 \%$ SAL $(n=4$, two to four MnPO sections per rat; $p=0.003$ ) groups. About half (54.4\%) of the Fos-positive cells in the MnPO were also CaMKIlapositive in the CTRL + CNO + ANG II rats. The percentages of Fos and CaMKIla-positive cells were $50.2 \%$ and $60.0 \%$ for CTRL + CNO + 0.9\% SAL and CTRL + VEH + $0.9 \%$ SAL rats, respectively. The increase in Fos and CaMKIla staining cells associated with ANG II was significantly attenuated in the $G_{i}$ DREADD + CNO + ANG II rats $(n=4$, two to four MnPO sections per rat; $p<0.001$; Fig. $7 B$ ). The percentage of Fos-positive cells that were also CaMKIla-positive was reduced to $6.5 \%$. This result indicates that over $90 \%$ of the remaining Fos-positive cells in the MnPO of rats injected with $G_{i}$ DREADD were not CaMKIla-positive cells, which is significantly greater than the other three treatment groups $\left(F_{(3,12)}=6.010, p=\right.$ 0.010 , one-way ANOVA; Tukey's post hoc analysis, all $p$ $<0.001$; Fig. 7B; Table 1). Since these cells do not appear to express CaMKIla, they would not have been transfected with the AAV vector used in this study. There was no significant difference in CaMKIla-positive neurons between any of the four groups $\left(F_{(3,12)}=0.0901, p=0.964\right.$, one-way ANOVA).

$G_{i}$ DREADD-mediated inhibition of the MnPO also influenced Fos staining associated with ANG II in regions connected to the MnPO $\left(G_{i}\right.$ DREADD + CNO + ANG II group: $n=6-10$, two to four sections per nucleus per rat; CTRL + CNO + ANG II group: $n=4-10$, two to four sections per nucleus per rat). In rats injected with the CTRL AAV vector and pretreated with CNO, ANG II significantly increased Fos staining in each region that we examined (Fig. 8). However, the effects of $G_{i}$ DREADD $\mathrm{MnPO}$ inhibition on ANG II induced Fos staining varied as a function of region. In the rats injected with $G_{i}$ DREADD, CNO pretreatment did not significantly affect ANG II induced Fos staining in SFO, OVLT, LH, or PVT (Fig. 8B).

In other regions, $G_{i}$ DREADD-mediated inhibition of the $\mathrm{MnPO}$ did influence Fos staining. In the SON, CNO pretreatment in rats injected in the MnPO with $G_{i}$ DREADD was associated with a significant decrease in ANG IIinduced Fos $\left(F_{(3,24)}=19.328, p<0.001\right.$, one-way ANOVA; Fig. $8 A, B)$. Fos staining in the SON of CTRL + CNO + ANG II rats was significantly higher as compared to all of the other groups (Tukey's post hoc analysis, all $p<0.001$; Fig. $8 A, B)$. Similar results were seen in the rostral ventrolateral medulla (RVLM) (Fig. 8A,B). ANG II injections significantly increased Fos staining in the CTRL $+\mathrm{CNO}+$ ANG II rats but not in the $\mathrm{G}_{\mathrm{i}}$ DREADD + CNO + ANG II rats $\left(F_{(3,16)}=29.480, p<0.001\right.$, one-way ANOVA).

In the PVN, CNO and ANG II significantly increased Fos staining in CTRL rats and there was a statistical trend for decreased Fos staining in the $G_{i}$ DREADD + CNO + ANG II as compared to CTRL + CNO + ANG II $\left(F_{(3,24)}=18.302\right.$, $p<0.001$, one-way ANOVA; Tukey's post hoc analysis, $p$ $=0.084)$. However, significant effects of $G_{i}$ DREADDmediated inhibition on Fos staining were observed in specific subregions of the PVN (Fig. 8C). For example, the posterior magnocellular (PM) part of PVN Fos staining was significantly increased by ANG II in CTRL rats while CNO significantly decreased Fos staining associated with ANG II in $G_{i}$ DREADD rats $\left(F_{(3,22)}=49.423, p<0.001\right.$, one-way ANOVA; Tukey's post hoc analysis, CTRL + CNO + ANG II; $p<0.001$ from all other groups). Fos staining in the PM of $G_{i}$ DREADD + CNO + ANG II rats was still significantly higher when compared to Fos stain- 


\section{A CTRL + VEH + 0.9\% SAL}

CTRL + CNO + 0.9\% SAL

\section{CTRL + CNO + ANG II}

$G_{i}$ DREADD + CNO + ANG II
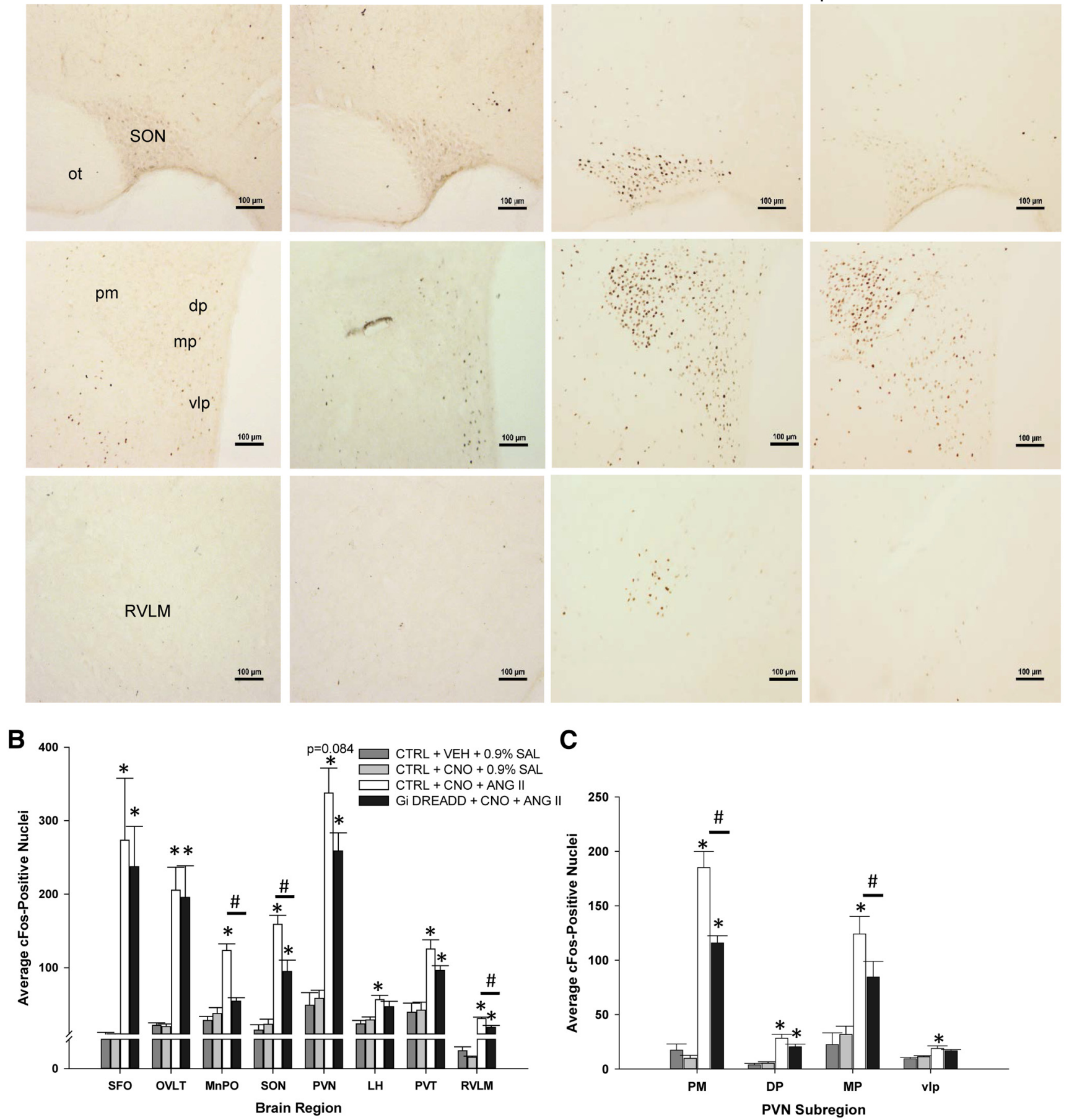

Figure 8. ANG II significantly increases Fos expression in regions downstream of the MnPO compared to controls and is blunted during $\mathrm{CNO}$-induced inhibition in $\mathrm{G}_{\mathrm{i}}$ DREADD-injected rats. A, Representative Fos staining in the SON (top row), PVN (middle row), and RVLM (bottom row) for each group tested. Scale bar, $100 \mu \mathrm{m}$. Supraoptic nucleus, SON; optic tract, ot: paraventricular nucleus, PVN; posterior magnocellular, PM; dorsal parvocellular, DP; medial parvocellular, MP; ventrolateral parvocellular, vlp; rostral ventral lateral medulla, RVLM. B, ANG II significantly increases Fos expression in the SFO, OVLT, PVN, and PVT but this increase was significantly attenuated during CNO-mediated inhibition in the MnPO, SON, LH, and RVLM. Subfornical organ, SFO; organum vasculosum of the lamina terminalis, OVLT; paraventricular thalamus, PVT; lateral hypothalamus, LH.C, ANG II significantly increases Fos staining in cardiovascular and neuroendocrine-regulating regions of the PVN, but is significantly attenuated by CNO-mediated inhibition in the PM and MP subregions; $* p<0.005$ compared to VEH (CTRL + VEH + 0.9\% SAL) and CNO (CTRL + CNO + 0.9\% $\mathrm{SAL})$ controls; $\# p<0.001 \mathrm{G}_{\mathrm{i}}$ DREADD-injected rats compared to control virus-injected rats treated with $C N O$ and $A N G$ II $\left(G_{i} D_{R E A D D}\right.$ + CNO + ANG II and CTRL + CNO + ANG II, respectively). Data are presented as mean and SEM. 
ing in rats pretreated with either CNO or VEH followed by $0.9 \%$ SAL (Tukey's post hoc analysis; $p=0.05$ and $p=$ 0.097 , respectively). DREADD-mediated inhibition of the $\mathrm{MnPO}$ influenced Fos staining in the medial parvocellular region (MP) of the PVN as well. In the MP part of the PVN, Fos staining associated with CNO and ANG II injections in CTRL rats was significantly greater than Fos staining observed in any of the other groups $\left(F_{(3,22)}=13.789, p<\right.$ 0.001 , one-way ANOVA; Tukey's post hoc analysis, all $p$ $<0.001)$. The Fos staining in the MP of $G_{i}$ DREADD + CNO + ANG II rats was not significantly different from the CTRL rats pretreated with either CNO or VEH followed by $0.9 \%$ SAL (Tukey's post hoc analysis; $p=0.097$ and $p=$ 0.050 , respectively). This suggested that in the MP region of the PVN, inhibition of the MnPO reduced ANG IIinduced Fos staining comparably to control levels. Similar results were observed in the ventrolateral parvocellular region (vlp). Fos staining was increased in CTRL + CNO + ANG II rats and this increase was significantly different from all of the other groups $\left(F_{(3,22)}=3.734, p=0.026\right.$, one-way ANOVA). Fos staining in the dorsal parvocellular part of the PVN was increased by ANG II $\left(F_{(3,22)}=11.903\right.$, $p<0.001$, one-way ANOVA) but CNO-induced inhibition did not significantly decrease it (CTRL + CNO + ANG II vs $\mathrm{G}_{i}$ DREADD + CNO + ANG II, $p=0.258$, Tukey's post hoc analysis).

\section{CNO-induced inhibition in the MnPO and Fos staining associated with $3 \%$ HTN}

In rats treated with the control vector, Fos staining in the MnPO was significantly increased by $3 \%$ HTN (CTRL $+\mathrm{VEH}+\mathrm{HTN}$ and CTRL + CNO + HTN; $F_{(3,16)}=4.982$, $p=0.002$, one-way ANOVA; Fig. $9 A, B)$. Half of the cells that were Fos-positive were also positive for CaMKIla (Table 2), suggesting that HTN affected cells that were not transfected with the vector.

Unlike our results with ANG II, Fos staining associated with $3 \% \mathrm{HTN}$ in the MnPO was not significantly decreased by $C N O$ pretreatment in $G_{i}$ DREADD rats (Gi DREADD + CNO + HTN). Fos staining in the MnPO of the $G_{i}$ DREADD + CNO + HTN group ( $n=6$ rats, two to four $\mathrm{MnPO}$ sections per rat) was significantly increased as compared to either of the CTRL groups injected with $0.9 \% \mathrm{SAL}$ (CTRL $+\mathrm{CNO}+0.9 \% \mathrm{SAL}, n=6$ rats, two to four MnPO sections per rat; CTRL + VEH + 0.9\% SAL, $n$ $=6$ rats, two to four MnPO sections per rat; $p=0.019$ and $p=0.006$, respectively). Fos staining in the $G_{i} D R E-$ $\mathrm{ADD}+\mathrm{CNO}+\mathrm{HTN}$ was not different from CTRL + CNO $+3 \%$ HTN rats ( $p=0.893$, Tukey's post hoc analysis).

In contrast, the number of Fos-positive cells that were also labeled for CaMKIla was significantly decreased (Table 2). As indicated above, half of the Fos-positive cells were CaMKIla-positive in the CTRL + CNO + 3\% HTN rats. In the $\mathrm{G}_{i}$ DREADD + CNO + 3\% HTN rats, only $5 \%$ of the Fos-positive cells were also positive for CaMKIla meaning $95 \%$ of the cells had a different phenotype and may have intrinsic osmotic sensitivity. There were no significant differences in the numbers CaMKIla-positive neurons between any of the four groups $\left(F_{(3,12)}=0.287, p\right.$ $=0.834$, one-way ANOVA; Table 2$)$. This suggests that the apparent lack of CNO inhibition on HTN-mediated Fos staining in the MnPO was due to activation of nonCaMKIla-expressing cells.

\section{CaMKIla neuron inhibition in the MnPO significantly decreases 3\% HTN-evoked effects in select downstream nuclei}

Analysis of Fos staining was performed in the same downstream nuclei as in the ANG II experiments above. While all of the regions except the SFO showed significant increases in Fos staining associated with the 3\% HTN injections, only the SON and LH were affected by $\mathrm{G}_{i}$ DREADD-mediated inhibition of the MnPO (Fig. 9). In the SONs of CTRL $+\mathrm{CNO}+3 \%$ HTN rats, Fos staining was significantly increased compared to all of the other groups $\left(F_{(3,16)}=52.418, p<0.001\right.$, one-way ANOVA; all $p<$ 0.001 , Tukey's post hoc analysis; Fig. $9 A, B)$. In the SONs of $G_{i}$ DREADD rats, CNO pretreatment significantly reduced Fos staining associated with $3 \%$ HTN as compared to CTRL + CNO $+3 \%$ HTN rats $(p=0.012$, Tukey's post hoc analysis) but did not reduce the staining to control levels (vs CTRL + VEH + 0.9\% SAL, $p<0.001$; vs CTRL + CNO $+0.9 \%$ SAL, $p<0.001$ ).

Similar results were observed in the $\mathrm{LH}$. Rats in the CTRL + CNO $+3 \%$ HTN group had significantly more Fos staining in the LH compared to the other three groups $\left(F_{(3,16)}=18.937, p<0.001\right.$, one-way ANOVA; all $p<$ 0.001 , Tukey's post hoc analysis; Fig. 9B). Fos staining in the $\mathrm{LH}$ of $\mathrm{G}_{\mathrm{i}}$ DREADD + CNO + 3\% HTN rats had significantly less Fos expression than the CTRL + CNO + $3 \%$ HTN-treated rats $p=0.001$, Tukey's post hoc analysis; Fig. $9 B$ ) and significantly higher than both the $0.9 \%$ SAL-treated groups (both $p<0.001$, Tukey's post hoc analysis; Fig. 9B). For the remaining regions (OVLT, PVN, PVT, and RVLM) Fos staining associated with CNO and $3 \%$ HTN was not different between the CTRL virus and $G_{i}$ DREADD-injected rats and both of these groups were significantly increased as compared to the two CTRL groups that received the $0.9 \% \mathrm{SAL}$ injection (Fig. 9A,B). This result was also observed in the PM and MP regions of the PVN (Fig. 9C), while 3\% HTN did not influence Fos staining in either the dorsal or vlp regions.

\section{Discussion}

These studies tested the role of putative excitatory $\mathrm{MnPO}$ neurons in behavioral and physiologic responses produced by peripheral administration of ANG II or 3\% HTN. The virally-mediated chemogenetic inhibition was employed in these studies to remotely and selectively reduce the activity of CaMKIla-expressing MnPO neuronal population in the least invasive manner. These experiments showed that acutely inhibiting the CaMKIlaexpressing neurons in the MnPO significantly attenuated water intake and vasopressin release produced by either ANG II or $3 \%$ HTN. The results, however, suggest several differences in how the MnPO contributes to these responses.

In previous studies of the lamina terminalis and its role in water consumption, the SFO and OVLT have been linked to body fluid homeostasis. Studies performed in sheep using electrolytic lesions placed along the ventral 


\section{A CTRL + CNO + 3\% HTN G DREADD + CNO + 3\% HTN}

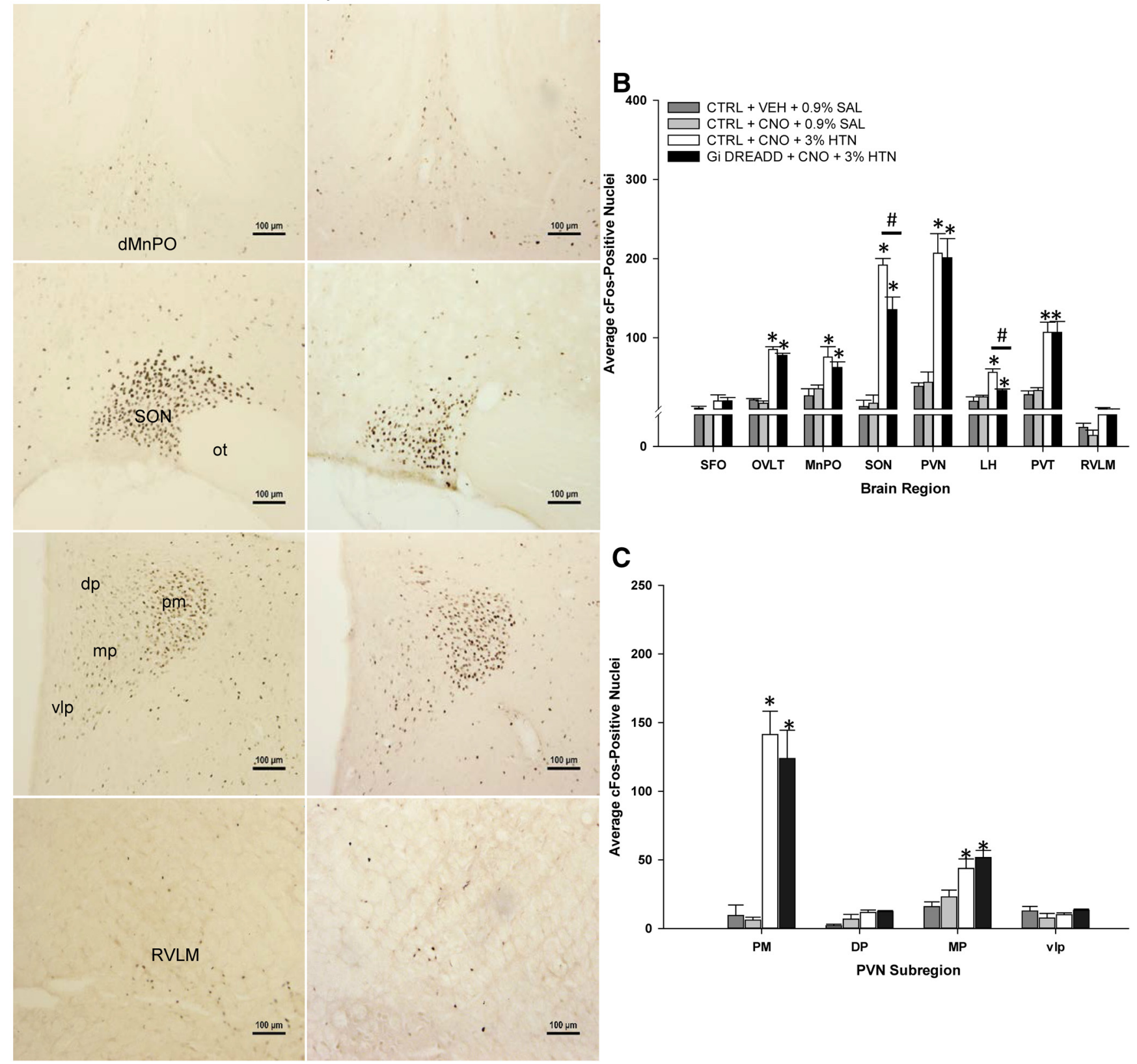

Figure 9. Hypertonic saline significantly increases Fos expression in the MnPO and in regions downstream, but is attenuated during CNO-induced inhibition in $\mathrm{G}_{\mathrm{i}}$ DREADD-injected rats. $\boldsymbol{A}$, Representative Fos staining in control virus-injected $(C T R L)$ rats $(n=6$, two to four sections per rat; left) and $\mathrm{G}_{\mathrm{i}}$ DREADD rats $(n=6$, two to four sections per rat; right), treated with CNO and $3 \% \mathrm{HTN}$; dMnPO (top row), SON (second row), PVN (third row), RVLM (bottom row). B, 3\% HTN significantly increases Fos expression in the OVLT, $\mathrm{MnPO}, \mathrm{PVN}$, and PVT, but this increase was significantly attenuated during CNO-mediated inhibition in the SON and LH. Scale bar, $100 \mu \mathrm{m}$. Subfornical organ, SFO; organum vasculosum of the lamina terminalis, OVLT; paraventricular thalamus, PVT; lateral hypothalamus, LH. C, 3\% HTN significantly increases Fos staining in cardiovascular and neuroendocrine-regulating regions of the PVN, the PM and MP subregions; $* p<0.005$ compared to VEH (CTRL + VEH + 0.9\% SAL) and CNO (CTRL + CNO + 0.9\% SAL) controls; $\# p<0.001 \mathrm{G}_{\mathrm{i}}$ DREADD rats compared to CTRL rats treated with CNO and $3 \% \mathrm{HTN}\left(\mathrm{G}_{\mathrm{i}}\right.$ DREADD + CNO + $3 \% \mathrm{HTN}$ and CTRL + CNO + 3\% HTN, respectively). Data are presented as mean and SEM.

lamina terminalis, including the OVLT, decreased water intake stimulated by cellular dehydration (McKinley et al., 1999). Chemogenetic activation of the CaMKIla neuronal phenotype in the SFO has also been shown to influence drinking behavior (Nation et al., 2016). Studies performed by Oka et al. (2015) showed that 48-h water deprivation induced Fos immunoreactivity and were able to identify that in the SFO all of the neurons expressing Fos colocalized with CaMKIIla and nNOS. The SFO and the OVLT both project to the $\mathrm{MnPO}$ as well as other regions involved in body fluid homeostasis (McKinley et al., 2004). Recent studies conducted by Augustine et al. (2018) im- 
Table 2. Colocalization of hypertonic saline-induced Fos expression with CaMKIla neurons in the MnPO

\begin{tabular}{|c|c|c|c|c|}
\hline Treatment & Total cFos + & Total CaMKIla+ & Total DL cells & $\%$ DL cFos + \\
\hline $\begin{array}{l}\text { Control } \\
\mathrm{CNO}+0.9 \% \mathrm{SAL}\end{array}$ & $16.1 \pm 3.7$ & $226.4 \pm 50.9$ & $8.6 \pm 2.2$ & $63.3 \pm 16.9 \%$ \\
\hline $\begin{array}{l}\text { Control } \\
\text { VEH + } 0.9 \% \text { SAL }\end{array}$ & $22.1 \pm 4.0$ & $202.9 \pm 47.3$ & $11.3 \pm 2.1$ & $74.6 \pm 1.3 \%$ \\
\hline $\begin{array}{l}\text { Control } \\
\text { CNO + } 3 \% \text { HTN }\end{array}$ & $74.4 \pm 17.6 *$ & $194.3 \pm 59.4$ & $30.0 \pm 8.3 * *$ & $49.5 \pm 16.7 \%$ \\
\hline $\mathrm{G}_{\mathrm{i}}$ DREADD CNO + 3\% HTN & $64.6 \pm 6.5 *$ & $165.4 \pm 22.1$ & $3.4 \pm 1.0$ & $5.4 \pm 1.9 \% * *$ \\
\hline
\end{tabular}

Hypertonic saline exposure significantly increases Fos expression in the MnPO, not only in CaMKIla-positive neurons, but also other neuronal phenotypes.

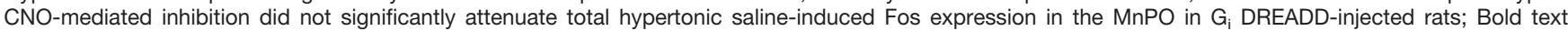
and $* p<0.050$ compared to vehicle controls; Bold text and $* * p<0.001$ compared to all groups. Data are presented as mean \pm SEM.

plicated the MnPO ${ }^{\text {NNOS }}$ neuronal phenotype in regulating drinking behavior stimulated by water deprivation and salt loading. The current study shows that CNO-induced inhibition of DREADD-transfected CaMKIla MnPO neurons significantly attenuates extracellular and cellular thirst. The result of the Fos studies indicate, however, that the homeostatic responses could involve different pathways from the MnPO.

These studies specifically targeted CaMKIla neurons in the MnPO, which as shown in the in situ hybridization studies, is highly co-localized with vGLUT2. This indicates that CaMKIla neurons in the MnPO are primarily glutamatergic. This is an important consideration since the MnPO has been shown to contain cells that can stimulate or inhibit water intake (Oka et al., 2015). The results of the current study extend the observations of Oka et al. (2015) by demonstrating that water intake related to extracellular and cellular dehydration is mediated by putative excitatory neurons in $\mathrm{MnPO}$.

The CaMKIla MnPO neurons transfected by the virus were sensitive to both ANG II and HTN-aCSF. The design of the experiments prevented us from being able to test the same cells with both ANG II and HTN-aCSF. Previous studies have defined a set of sodium sensitive MnPO neurons that likely contribute to body fluid balance (Grob et al., 2004) and additionally characterized ANG IIsensitive MnPO neurons in earlier experiments, consistent with our findings (Bai and Renaud, 1998). The relationship between ANG II and HTN-aCSF in the regulation of the $\mathrm{MnPO}$ neurons will be the focus of future investigations.

The CNO-mediated inhibition of CaMKIla-positive neurons in the MnPO completely blocked ANG II-induced excitatory responses. However, during osmotic challenges, CNO-mediated inhibition was only able to significantly attenuate osmotic-induced excitatory response, in contrast to the complete inhibition of ANG II-induced excitation. This is consistent with studies conducted by Grob et al. (2004) describing a specific MnPO neuronal phenotype with intrinsic sodium sensitivity. Recordings performed in the current experiments also verify that $G_{i}$ DREADDx neurons, or MnPO neurons that do not express CaMKIla, are also sensitive to ANG II and osmotic challenges (Grob et al., 2004).

Results from these Fos studies further support the electrophysiology findings and suggest that ANG II and 3\% HTN mediated responses could involve different populations of CaMKIlla-expressing MnPO neurons. Fos immunohistochemistry and its co-localization with CaMKIla in the $\mathrm{MnPO}$ was analyzed. Neurons in the MnPO both expressing CaMKIla and Fos were significantly elevated in groups injected with the control virus and administered ANG II or 3\% HTN. During DREADD-mediated inhibition using $\mathrm{CNO}$, however, there were significantly reduced numbers of CaMKIla-positive cells that were also positive for Fos in the MnPO. While this led to a significant decrease in the total number of Fos-positive cells associated with ANG II, the overall decrease in the MnPO was not significant after hypertonic saline. This could be related to the electrophysiology data showing that CNO did not completely block the hyperosmotically-stimulated excitation as strongly as the ANG II responses in the $G_{i}$ DREADD-transfected neurons.

The differences observed for Fos staining in the MnPO could be related to the nonspecific effects of the hypertonic saline injections. While hypertonic saline is widely used as an osmotic stimulus, Fos staining associated with its administration may be a result of visceral pain related to the route of injection, and therefore, not specific. However, a study conducted by Xu et al. (2003) compared Fos staining produced by intraperitoneal versus intravenous administration of $2.0 \mathrm{M} \mathrm{NaCl}$ in several of the regions included in the current study (MnPO, OVLT, PVN, and SON). They concluded that the increases in Fos staining observed in areas such as the MnPO were related osmotic stimulation and fluid balance and not nociception. Similar conclusions were made about Fos staining in the $\mathrm{LH}$ produced intraperitoneal administration of $1.5 \mathrm{M} \mathrm{NaCl}$ (Pirnik et al., 2004). It should be noted that the concentration of $\mathrm{NaCl}$ used in the current study are approximately four times lower than the concentration used by $\mathrm{Xu}$ et al. (2003) and that this study used subcutaneous injections to avoid producing visceral pain associated with intraperitoneal injections. Therefore, the Fos staining produced by hypertonic saline is more likely related to osmotic stimulation but the contribution of nociceptive stimulation at the injection site cannot be ruled out.

There were also observed differences in Fos staining in several other regions that receive projections from the $\mathrm{MnPO}$ that were stimulus dependent. In rats administered ANG II, CNO-induced inhibition of $G_{i}$ DREADD was associated with significant decreases in Fos staining in the SON, PVN, and RVLM. When rats were injected with hypertonic saline, $G_{i}$ DREADD mediated inhibition of the $\mathrm{MnPO}$ significantly attenuated Fos staining in the SON and LH but not the PVN or RVLM. The central effects of ANG II and hypertonic saline are mediated by CVOs (Fer- 
guson, 2014). These regions project not only to the MnPO but also to the SON, PVN, and LH (McKinley et al., 2004, 2015; Ferguson, 2014). This creates a series of redundant pathways from the lamina terminalis to regions contributing to hormone release and water intake. Our results suggest that, despite these possible redundancies, the $\mathrm{MnPO}$ significantly contributes to the activation of SON, $\mathrm{PVN}$, and $\mathrm{LH}$ in a stimulus-dependent manner.

As shown in these studies, $G_{i}$ DREADD inhibition of the $\mathrm{MnPO}$ was associated with significant decreases in Fos staining in the SON after either ANG II or 3\% HTN. These results are consistent with observed reductions in AVP release associated with $G_{i}$ DREADD inhibition of the MnPO during ANG II or 3\% HTN exposure, with ANG II inducing a greater release of AVP than 3\% HTN between groups. This may be due to how each stimulus induces its effects in the local circuitry. In ANG II-treated rats, $G_{i}$ DREADD inhibition of the MnPO reduced Fos staining in the PVN and RVLM, while these regions were not influenced by MnPO inhibition in hypertonic saline-treated rats. Fos staining in the $\mathrm{LH}$ that was associated with hypertonic saline was significantly decreased by $G_{i} D R E-$ ADD inhibition. Recent studies suggest that the PVN, PVT, and $\mathrm{LH}$ receive projections from the $\mathrm{MnPO}$ that are related to water intake (Leib et al., 2017). Results from the current study are generally consistent with these findings and suggest that these pathways might be differentially regulated by extracellular dehydration versus cellular dehydration (Fitzsimons, 1972). Differences in MnPO neurons that contribute to extracellular versus cellular was first proposed based on the results of studies using excitotoxins (Cunningham et al., 1991). The current results provide evidence that MnPO neurons mediating water intake associated with extracellular dehydration project to the PVN while MnPO neurons participating in cellular thirst project to the $\mathrm{LH}$. These data also suggest that there are MnPO neurons participating in both extracellular and cellular dehydration that project to the SON.

Findings from the current study also demonstrate that Fos expression was significantly decreased in the RVLM during ANG II but not hypertonic saline exposure during $G_{i}$ DREADD-mediated inhibition. The RVLM contains sympathetic motor neurons that contribute to blood pressure regulation. These results suggest that the MnPO may contribute to increases in sympathetic tone related to activation of the renin-angiotensin system but not high salt. This is consistent with previous studies on the role of the lamina terminalis in ANG II-dependent models of hypertension (Ployngam and Collister, 2007; Collister et al., 2014; Shell et al., 2016). The decreased Fos staining in the RVLM could be related to the decreased Fos expression observed in the PVN, which has also been linked to hypertension (Llewellyn et al., 2012; Basting et al., 2018). Lesions of the AV3V have been shown prevent or reverse several ANG II dependent models of hypertension (Brody et al., 1978). The results of the current study suggest that CaMKIla neurons in the MnPO that project to the PVN could contribute to this aspect of AV3V function.

Interestingly, ANG II was much more effective at inducing differential Fos expression, water intake, and AVP release compared to $3 \%$ HTN. Studies by Fitzsimons showed that water consumption associated with hypovolemic dehydration can result in a greater volume of water intake as compared to cellular dehydration of similar magnitude (Fitzsimons, 1998). While it is difficult to determine the equivalence of extracellular versus cellular dehydration, the differences in the magnitude of the response could be related to the position of the doses used in their respective dose-response curves.

It is important to note how increases in arterial pressure associated with ANG II may alter its dipsogenic effects. Early studies addressing ANG II in relation to arterial pressure and thirst have been conducted. Rettig and Johnson (1986) studied the role of ANG II, among other stimuli, on drinking in sinaortic denervated rat models to hypovolemia, and found no effect on water intake. In other studies conducted by Evered et al. (1988), the interaction between the dipsogenic and pressor effects of ANG II at various concentrations were tested using diazoxide, a vasodilator, to counteract the increases in blood pressure (Evered et al., 1988). It was concluded from these studies that intravenous infusions of ANG II in water-replete rats stimulate thirst in a dose-dependent manner, but water intake may be attenuated due to the concurrent increase in blood pressure. The rise in arterial pressure may also produce increases in urinary water and solute excretion, resulting in dehydration that could eventually contribute to thirst as well (Evered et al., 1988). Most physiologic situations characterized by activation of the renin-angiotensin system include hypovolemia and hypotension. In this context, the increase in blood pressure produced by ANG II may allow the rats to be behaviorally competent enough to ingest water (Evered et al., 1988).

In summary, these studies used ANG II and hypertonic saline injections to simulate aspects of extracellular and cellular dehydration to better understand the contribution of the MnPO to the integrative physiology of body fluid homeostasis. Both of these stimuli act through CVOs to affect the MnPO, Additionally, hypertonic saline can directly influence the activity of sodium sensitive MnPO neurons. The chemogenetic inhibition of putative excitatory $\mathrm{MnPO}$ neurons inhibited vasopressin release and drinking behavior produced by either ANG II or hypertonic saline. In contrast, the effects of inhibiting CaMKIla MnPO neurons was associated with different patterns of Fos staining. Fos staining in the SON, PVN, and RVLM associated with ANG II injections was significantly decreased by chemogenetic inhibition of the MnPO. After injections of hypertonic saline, MnPO inhibition affected the $\mathrm{LH}$ and SON. Thus, extracellular and cellular dehydration appear to influence different populations of CaMKIla-expressing $\mathrm{MnPO}$ neurons based on their afferent projections but can result in converging behavioral outcomes.

\section{References}

Adler SM, Verbalis JG (2006) Disorders of body water homeostasis in critical illness. Endocrinol Metab Clin North Am 35:873-894.

Allen WE, DeNardo LA, Chen MZ, Liu CD, Loh KM, Fenno LE, Ramakrishnan C, Deisseroth K, Luo L (2017) Thirst-associated preoptic neurons encode an aversive motivational drive. Science $357: 1149-1155$ 
Augustine V, Gokce SK, Lee S, Wang B, Davidson TJ, Reimann F, Gribble F, Deisseroth K, Lois C, Oka Y (2018) Hierarchical neural architecture underlying thirst regulation. Nature 555:204-209.

Bai D, Renaud LP (1998) ANG II AT1 receptors induce depolarization and inward current in rat median preoptic neurons in vitro. Am J Physiol 275:R632-R639.

Basting T, Xu J, Mukerjee S, Epling J, Fuchs R, Sriramula S, Lazartigues $E$ (2018) Glutamatergic neurons of the paraventricular nucleus are critical contributors to the development of neurogenic hypertension. J Physiol 596:6235-6248.

Bourque CW (2008) Central mechanisms of osmosensation and systemic osmoregulation. Nat Rev Neurosci 9:519-531.

Brody MJ, Fink GD, Buggy J, Haywood JR, Gordon FJ, Johnson AK (1978) The role of the anteroventral third ventricle (AV3V) region in experimental hypertension. Circ Res 43:I2-I13.

Collister JP, Bellrichard M, Drebes D, Nahey D, Tian J, Zimmerman MC (2014) Over-expression of copper/zinc superoxide dismutase in the median preoptic nucleus attenuates chronic angiotensin II-induced hypertension in the rat. Int J Mol Sci 15:22203-22213.

Cunningham JT, Sullivan MJ, Edwards GL, Farinpour R, Beltz TG, Johnson AK (1991) Dissociation of experimentally induced drinking behavior by ibotenate injection into the median preoptic nucleus. Brain Res 554:153-158.

Cunningham JT, Beltz T, Johnson RF, Johnson AK (1992) The effects of ibotenate lesions of the median preoptic nucleus on experimentally-induced and circadian drinking behavior in rats. Brain Res 580:325-330.

Dampney RA (2016) Central neural control of the cardiovascular system: current perspectives. Adv Physiol Educ 40:283-296.

Evered MD, Robinson MM, Rose PA (1988) Effect of arterial pressure on drinking and urinary responses to angiotensin II. Am J Physiol 254:R69-R74.

Farmer GE, Balapattabi K, Bachelor ME, Little JT, Cunningham JT (2018) AT1a influences GABAa mediated inhibition through the regulation of KCC2 expression. Am J Physiol Regul Integr Comp Physiol 315:R972-R982.

Ferguson AV (2014) Frontiers in neuroscience circumventricular organs: integrators of circulating signals controlling hydration, energy balance, and immune function. In: Neurobiology of body fluid homeostasis: transduction and integration (De Luca LA Jr, Menani JV, Johnson AK, eds). Boca Raton, FL: CRC Press/Taylor \& Francis.

Fitzsimons JT (1972) Thirst. Physiol Rev 52:468-561.

Fitzsimons JT (1998) Angiotensin, thirst, and sodium appetite. Physiol Rev 78:583-686.

Gardiner TW, Stricker EM (1985) Hyperdipsia in rats after electrolytic lesions of nucleus medianus. Am J Physiol 248:R214-R223.

Gardiner TW, Verbalis JG, Stricker EM (1985) Impaired secretion of vasopressin and oxytocin in rats after lesions of nucleus medianus. Am J Physiol 249:R681-R688.

Grindstaff RJ, Grindstaff RR, Sullivan MJ, Cunningham JT (2000) Role of the locus ceruleus in baroreceptor regulation of supraoptic vasopressin neurons in the rat. Am J Physiol Regul Integr Comp Physiol 279:R306-R319.

Grob M, Drolet G, Mouginot D (2004) Specific Na+ sensors are functionally expressed in a neuronal population of the median preoptic nucleus of the rat. J Neurosci 24:3974-3984.
Hoffman GE, Smith MS, Fitzsimmons MD (1992) Detecting steroidal effects on immediate early gene expression in the hypothalamus. Neuroprotocols 1:55-66.

Johnson AK, Cunningham JT, Thunhorst RL (1996) Integrative role of the lamina terminalis in the regulation of cardiovascular and body fluid homeostasis. Clin Exp Pharmacol Physiol 23:183-191.

Leib DE, Zimmerman CA, Poormoghaddam A, Huey EL, Ahn JS, Lin YC, Tan CL, Chen Y, Knight ZA (2017) The forebrain thirst circuit drives drinking through negative reinforcement. Neuron 96:12721281.e4

Llewellyn T, Zheng H, Liu X, Xu B, Patel KP (2012) Median preoptic nucleus and subfornical organ drive renal sympathetic nerve activity via a glutamatergic mechanism within the paraventricular nucleus. Am J Physiol Regul Integr Comp Physiol 302:R424R432.

McKinley MJ, Mathai ML, Pennington G, Rundgren M, Vivas L (1999) Effect of individual or combined ablation of the nuclear groups of the lamina terminalis on water drinking in sheep. Am J Physiol 276:R673-R683.

McKinley MJ, Mathai ML, McAllen RM, McClear RC, Miselis RR, Pennington GL, Vivas L, Wade JD, Oldfield BJ (2004) Vasopressin secretion: osmotic and hormonal regulation by the lamina terminalis. J Neuroendocrinol 16:340-347.

McKinley MJ, Yao ST, Uschakov A, McAllen RM, Rundgren M, Martelli D (2015) The median preoptic nucleus: front and centre for the regulation of body fluid, sodium, temperature, sleep and cardiovascular homeostasis. Acta Physiol (Oxf) 214:8-32.

Mecawi Ade S, Ruginsk SG, Elias LL, Varanda WA, AntunesRodrigues J (2015) Neuroendocrine regulation of hydromineral homeostasis. Compr Physiol 5:1465-1516.

Miselis RR (1982) The subfornical organ's neural connections and their role in water balance. Peptides 3:501-502.

Nation HL, Nicoleau M, Kinsman BJ, Browning KN, Stocker SD (2016) DREADD-induced activation of subfornical organ neurons stimulates thirst and salt appetite. J Neurophysiol 115:3123-3129.

Oka Y, Ye M, Zuker CS (2015) Thirst driving and suppressing signals encoded by distinct neural populations in the brain. Nature 520 : 349-352.

Pirnik Z, Mravec B, Kiss A (2004) Fos protein expression in mouse hypothalamic paraventricular (PVN) and supraoptic (SON) nuclei upon osmotic stimulus: colocalization with vasopressin, oxytocin, and tyrosine hydroxylase. Neurochem Int 45:597-607.

Ployngam T, Collister JP (2007) An intact median preoptic nucleus is necessary for chronic angiotensin II-induced hypertension. Brain Res 1162:69-75.

Rettig R, Johnson AK (1986) Aortic baroreceptor deafferentation diminishes saline-induced drinking in rats. Brain Res 370:29-37.

Shell B, Faulk K, Cunningham JT (2016) Neural control of blood pressure in chronic intermittent hypoxia. Curr Hypertens Rep 18: 19.

Smith JA, Wang L, Hiller H, Taylor CT, de Kloet AD, Krause EG (2014) Acute hypernatremia promotes anxiolysis and attenuates stressinduced activation of the hypothalamic-pituitary-adrenal axis in male mice. Physiol Behav 136:91-96.

Xu Z, Torday J, Yao J (2003) Functional and anatomic relationship between cholinergic neurons in the median preoptic nucleus and the supraoptic cells. Brain Res 964:171-178. 